\title{
Genome-wide identification of drought- responsive microRNAs in two sets of Malus from interspecific hybrid progenies
}

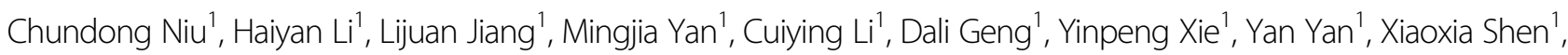
Pengxiang Chen ${ }^{1}$, Jun Dong ${ }^{1}$, Fengwang $\mathrm{Ma}^{1}$ and Qingmei Guan ${ }^{1}$

\begin{abstract}
Drought stress can negatively impact apple fruit quality and yield. Apple microRNAs (miRNAs) participate in apple tree and fruit development, as well as in biotic stress tolerance; however, it is largely unknown whether these molecules are involved in the drought response. To identify drought-responsive miRNAs in Malus, we first examined the drought stress tolerance of ten $F_{1}$ progenies of R3 $(M$. $\times$ domestica) $\times M$. sieversii. We performed Illumina sequencing on pooled total RNA from both drought-tolerant and drought-sensitive plants. The sequencing results identified a total of 206 known miRNAs and 253 candidate novel miRNAs from drought-tolerant plants and drought-sensitive plants under control or drought conditions. We identified 67 miRNAs that were differentially expressed in drought-tolerant plants compared with drought-sensitive plants under drought conditions. Under drought stress, 61 and 35 miRNAs were differentially expressed in drought-tolerant and drought-sensitive plants, respectively. We determined the expression levels of seven out of eight miRNAs by stem-loop qPCR analysis. We also predicted the target genes of all differentially expressed miRNAs and identified the expression of some genes. Gene Ontology analyses indicated that the target genes were mainly involved in stimulus response and cellular and metabolic processes. Finally, we confirmed roles of two miRNAs in apple response to mannitol. Our results reveal candidate miRNAs and their associated mRNAs that could be targeted for improving drought tolerance in Malus species, thus providing a foundation for understanding the molecular networks involved in the response of apple trees to drought stress.
\end{abstract}

\section{Introduction}

MicroRNAs (miRNAs) are 20-24 nt endogenous small RNAs that silence or downregulate gene expression at the transcriptional and posttranscriptional levels by targeting mRNAs through imperfect sequence complementarity ${ }^{1-3}$. Typically, plant miRNAs are cleaved from hairpin loop precursors by DICER-like $1^{4}$. Increasing evidence suggests that miRNAs are important regulators in various plant processes, such as development ${ }^{5}$ and both biotic and abiotic stress responses ${ }^{6}$.

\footnotetext{
Correspondence: Qingmei Guan (qguan@nwsuaf.edu.cn)

${ }^{1}$ State Key Laboratory of Crop Stress Biology for Arid Areas/Shaanxi Key Laboratory of Apple, College of Horticulture, Northwest A\&F University, Yangling, Shaanxi 712100, China

These authors contributed equally: Chundong Niu, Haiyan Li
}

A variety of approaches, such as northern blotting, cDNA microarrays, and small RNA high-throughput sequencing, have identified miRNA expression patterns in response to drought in Arabidopsis $^{7-9}$, rice $^{10-12}$, Populus trichocarpa ${ }^{13,14}$, tomato ${ }^{15}$, and cotton ${ }^{16}$. In Arabidopsis, northern blotting methods have revealed miRNAs that are responsible for drought-responsiveness. For example, miR169 is downregulated by drought stress 9 . A microarray platform identified 30 miRNAs in rice leaves that are significantly up- or downregulated in response to drought stress $^{10}$, whereas small RNA high-throughput sequencing identified 688 and 155 miRNAs differentially expressed under drought and salt stress in tomatoes ${ }^{15}$ and $\operatorname{cotton}^{16}$, respectively. Notably, in different plant species, identical miRNA sequences can have different expression

\section{(c) The Author(s) 2019}

(c) (i) Open Access This article is licensed under a Creative Commons Attribution 4.0 International License, which permits use, sharing, adaptation, distribution and reproduction c. in any medium or format, as long as you give appropriate credit to the original author(s) and the source, provide a link to the Creative Commons license, and indicate if changes were made. The images or other third party material in this article are included in the article's Creative Commons license, unless indicated otherwise in a credit line to the material. If material is not included in the article's Creative Commons license and your intended use is not permitted by statutory regulation or exceeds the permitted use, you will need to obtain permission directly from the copyright holder. To view a copy of this license, visit http://creativecommons.org/licenses/by/4.0/. 
patterns in response to drought. For example, miR408 is downregulated in Arabidopsis ${ }^{17}$ but upregulated in Medicago truncatula ${ }^{18}$ under drought conditions.

The regulation of miRNAs by drought is multifactorial and manifests across a range of physiological and biochemical mechanisms ${ }^{19}$. Physiological changes, such as early flowering, can improve plant drought resistance ${ }^{20}$. The SQUAMOSA promoter binding protein-like (SPL) transcription factor plays an important role in plant phase transitions and in tissue and architecture development ${ }^{21}$, such as the flowering process. Accordingly, high levels of miR156, which targets the SPL transcription factor, delay flowering time in Arabidopsis $^{22}$. Biological processes, such as auxin signaling, ABA-mediated regulation, osmoprotectant biosynthesis, and antioxidant scavenging, also modulate miRNA responses to drought ${ }^{23}$. In Arabidopsis, ABA treatment induces the expression of miR393, miR397b, and miR402 ${ }^{7}$. Silencing mitogen-activated protein kinase, a target of miR168, impairs ABA and hydrogen peroxide $\left(\mathrm{H}_{2} \mathrm{O}_{2}\right)$ signaling, resulting in reduced drought tolerance in the tomato plant ${ }^{24}$.

Apple (Malus $\times$ domestica Borkh.) is one of the most commercially important tree fruits worldwide and is mainly propagated through grafting; however, apple trees are often damaged by frequent environmental stressors, such as drought. Drought stress negatively affects fruit quality and yield, and even orchard ecosystem integrity, in some cases ${ }^{25}$. Therefore, it is a high priority to understand the molecular mechanisms orchestrating the response of apple to drought. To date, numerous miRNAs have been identified in apples and associated with either the development of apple trees ${ }^{26,27}$ or fruit ${ }^{28}$, or biotic stress responses ${ }^{29-31}$ but not with drought responses.

Malus sieversii is a widely used drought-resistant vigorous rootstock in northwest China ${ }^{32}$, whereas R3 is a promising dwarfing rootstock for apple. However, R3 is drought sensitive. To develop drought-tolerant dwarfing rootstocks for apple, we performed interspecific hybridization between R3 and M. sieversii. This approach has been used for breeding disease-resistant apples ${ }^{33}$.

To identify drought-responsive apple miRNAs, we first assessed the drought tolerance of ten $F_{1}$ progenies of R3 $(M . \times$ domestica $) \times M$. sieversii and then conducted small RNA sequencing (RNA-seq) on selected drought-tolerant and drought-sensitive plants under control or drought conditions. We provide basic information describing how miRNAs in Malus respond to drought stress and this information is urgently needed to facilitate drought tolerance in Malus species by genetic engineering methods.

\section{Materials and methods}

Plant materials and drought stress treatments

All plants were grown at Northwest A\&F University, Yangling, China $\left(34^{\circ} 20^{\prime} \mathrm{N}, 108^{\circ} 24^{\prime} \mathrm{E}\right)$.
In 2014, 500 interspecific crossed $F_{1}$ progenies of R3 $(M . \times$ domestica, a dwarfing rootstock with a droughtsensitive phenotype) $\times M$. sieversii (a vigorous rootstock with a drought-tolerant phenotype) were grown for 4 months in pots containing a local 5:1 (v:v) mixture of loess and sand. We then imposed a drought treatment on the plants for 7 days in mid-July 2014, with mostly sunny weather conditions and a mean high temperature of $34^{\circ} \mathrm{C}$. To avoid the death of $F_{1}$ plants due to drought stress, all $F_{1}$ plants were treated with relatively mild drought conditions. At the end of the drought treatment (where soil moisture content was $\sim 40 \%$ ), we evaluated the leaf wilting rates (the number of wilting leaves among total leaves in one tree). The wilted leaves were dehydrated and could not be recovered by watering. Plants with wilting rates $>50 \%$ were classified as drought-sensitive plants.

In 2015, 20 progenies from drought-sensitive and drought-tolerant plants were propagated through tissue culture. Briefly, the buds were disinfected with $75 \%$ alcohol, followed by $0.10 \%$ mercuric chloride before being placed on Murashige-Skoog (MS) medium supplemented with naphthyl acetic acid, polyvinylpolypyrrolidone, 6benzylaminopurine (6-BA), indole butyric acid, and sugar. Tissue-cultured $\mathrm{F}_{1}$ progenies were propagated and rooted before transplantation to a greenhouse in pots containing a local 5:1 (v:v) mixture of loess and sand. After removing the weak plants, we imposed a drought stress treatment in 2016 on the remaining plants to further confirm phenotypes under drought stress (Table 1). We began treating 10-month-old $F_{1}$ progenies with drought stress on 15 July 2016, with mostly cloudy weather conditions and a mean high temperature of $31^{\circ} \mathrm{C}$. Before drought treatment, these plants were all watered normally ( 1 liter per pot every 2 days). During drought treatment, the control (each progeny has three trees for control) treatment groups were watered every day to ensure a soil moisture content of $70 \%$. The drought treatment groups were weighed every day and the soil moisture content was calculated. After 12 days of severe drought treatment (where soil moisture content was $\sim 30 \%$ ), we recorded the wilting rate (trees with wilting rates $>50 \%$ were defined as wilting trees and the proportion of wilting trees among the total trees in each $F_{1}$ progeny was defined as the wilting rate) of each $F_{1}$ progeny. All plants were subsequently re-irrigated and grown for an additional 30 days and we scored the new growth leaves in each $F_{1}$ progeny as a recovery rate. Plants with recovery rates (in one progeny, the ratio of plants with new growth leaves to total plants) $<50 \%$ were classified as drought-sensitive plants and those with recovery rates $>50 \%$ were classified as drought-tolerant plants. Ultimately, we validated four drought-sensitive and six drought-tolerant plants from our drought tolerance assessment in 2014 and our determination of wilting and recovery rates in 2016. 
Table 1 Wilting rate after drought treatment and recovery rate after rehydration

\begin{tabular}{|c|c|c|c|c|c|c|}
\hline & \multirow[t]{2}{*}{ Line number } & \multirow{2}{*}{$\begin{array}{l}\text { Total number } \\
\text { of trees }\end{array}$} & \multicolumn{2}{|l|}{ Drought } & \multicolumn{2}{|l|}{ Rehydration } \\
\hline & & & $\begin{array}{l}\text { Number of } \\
\text { wilting trees }\end{array}$ & $\begin{array}{l}\text { Wilting } \\
\text { rate }(\%)\end{array}$ & $\begin{array}{l}\text { Number of } \\
\text { recovered trees }\end{array}$ & $\begin{array}{l}\text { Recovery } \\
\text { rate (\%) }\end{array}$ \\
\hline \multirow[t]{6}{*}{ Drought-tolerant plants } & $0-293$ & 10 & 7 & 70 & 7 & 70 \\
\hline & $0-106$ & 10 & 6 & 60 & 10 & 100 \\
\hline & $0-334$ & 9 & 5 & 56 & 9 & 100 \\
\hline & $7-275$ & 9 & 4 & 44 & 5 & 56 \\
\hline & $8-134$ & 9 & 7 & 78 & 8 & 89 \\
\hline & $0-330$ & 7 & 1 & 14 & 7 & 100 \\
\hline \multirow[t]{4}{*}{ Drought- sensitive plants } & $0-297$ & 5 & 5 & 100 & 2 & 40 \\
\hline & $0-131$ & 8 & 8 & 100 & 2 & 25 \\
\hline & $1-338$ & 5 & 4 & 80 & 1 & 20 \\
\hline & $8-11$ & 3 & 3 & 100 & 1 & 33 \\
\hline
\end{tabular}

Wilting rate means the proportion of wilting trees among the total trees in each $F_{1}$ progeny

\section{Constructing small RNA libraries for high-throughput sequencing}

In 2016, leaf samples were collected from droughtsensitive and drought-tolerant plants after drought treatment for 6 days, when the soil moisture content was between $40 \%$ and $50 \%$. We collected three leaves per plant (the 3rd, 4th, and 5th leaves from the top of a branch) from each tree under control (each progeny had three trees) or drought treatment (each progeny had at least six trees), except for $F_{1}$ progenies $0-297,1-338$, and 8-11. $F_{1}$ progenies $0-297,1-338$, and $8-11$ had 3-5 plants for drought treatment or control. We divided the leaves from each $F_{1}$ progeny into three biological replicates and each biological replicate contained at least one tree either under drought or control treatment. For each $F_{1}$ progeny biological replicate under control or drought treatment, total RNA was extracted using the miRcute miRNA Isolation Kit (TIANGEN, Beijing, China). Total RNA from drought-tolerant and drought-sensitive plants was pooled separately and used in the small RNA library construction. Specifically, equal amounts of total RNA from each progeny with the same phenotype were pooled as a single biological replicate. Three replicates were included for each control and drought-treated sample. RNA degradation and contamination were monitored on $1 \%$ agarose gels and RNA integrity was assayed by the Agilent Bioanalyzer 2100 System (Agilent Technologies, CA, USA).

A total of $3 \mu \mathrm{g}$ of total RNA per pooled sample was used for the small RNA library construction using the NEBNext ${ }^{\oplus}$ Multiplex Small RNA Library Prep Set for Illumina ${ }^{\oplus}$ (NEB, MA, USA). A total of 12 libraries were constructed, including three drought-sensitive plants under control conditions (S-C), three drought-tolerant plants under control conditions (T-C), three drought-sensitive plants under drought-treated conditions (S-D), and three drought-tolerant plants under drought-treated conditions (T-D). Then, we performed single-end sequencing (50 bp) on an Illumina Hiseq 2500 platform (Illumina, CA, USA) and generated 20 million reads per sample at Novogene Bioinformatics Institute (Novogene, Beijing, China). The small RNA-seq data were deposited to NCBI under accession number SRP110881.

\section{Identification and analysis of known and novel miRNAs}

Raw reads were cleaned by removing the reads containing poly $\mathrm{A} / \mathrm{T} / \mathrm{G} / \mathrm{C}$, reads with $5^{\prime}$ adapter contaminants, reads without the $3^{\prime}$ adapter or the insert tag, and low-quality reads. Q20, Q30, and the GC content of the raw data were calculated to analyze the quality of the small RNA libraries and then a certain length range of clean reads was selected for all downstream analyses. The length distribution from 18 to 30 nt was analyzed for clean reads and the reads ranging from 18 to $30 \mathrm{nt}$ were mapped to the domesticated apple reference genome sequence (https://www.ncbi.nlm.nih.gov/genome/?term $=$ malus) by using Bowtie $2^{34}$, with no mismatches allowed. Unmapped sequences were removed from the data.

The alignment of known miRNAs, prediction of novel miRNAs, miRNA family analysis, miRNA counts and normalization, and differential expression analysis were performed according to $\mathrm{Li}$ et $\mathrm{al}^{35}$. To predict novel miRNAs, known miRNA tags obtained by mirdeep $2^{36}$ and srna-tools-cli ${ }^{37}$ were removed. To map every unique small RNA to only one annotation, we filtered out tags of ribosomal RNA (rRNA), transfer RNA (tRNA), small 
nuclear ribonucleic acid (snRNA), and small nucleolar RNA (snoRNA) with the Rfam database (http://rfam. xfam.org/), repeat sequences with the annotation of Malus, natural antisense transcript small interfering RNA (NAT-siRNA) with PlantNATsDB (http://bis.zju.edu.cn/ pnatdb/), and genes (exon and intron were included to exclude mRNA degradation fragments) with the gene annotation of Malus. Finally, the remaining sRNA tags were used to predict novel miRNAs by miREvo ${ }^{38}$ and mirdeep $2^{36}$, and the read counts were counted as described $^{35}$. For the miRNA family analysis, miFam.dat (http://www.mirbase.org/ftp.shtml) was used for known miRNAs and Rfam (http://rfam.sanger.ac.uk/search/) was used for novel miRNAs. We normalized the abundance of miRNAs in each library using transcripts per million ${ }^{39}$. To obtain drought-responsive miRNAs, the miRNA expression under drought treatment was compared with that under control conditions in drought-tolerant or droughtsensitive plants. To identify drought tolerance-related miRNAs, the miRNA expression in drought-tolerant plants was compared with that in drought-sensitive plants under drought treatment or control conditions. The DESeq R package (1.8.3) was used for the differential expression analysis and the threshold for significant differential expression was $P<0.05$. To draw heatmaps and Venn diagrams, we used OmicShare tools (http://www. omicshare.com/tools).

\section{Target prediction and GO enrichment analysis}

To target candidate mRNAs for drought improvement in apple rootstocks, we selected differentially expressed miRNAs for further study. The target prediction was made through the psRNATarget server (http://plantgrn. noble.org/psRNATarget/) with default parameters of schema V1 (2011 release) ${ }^{40}$ using the differentially expressed miRNAs and domesticated apple RNA database (https://www.ncbi.nlm.nih.gov/genome/?term = malus).

To further evaluate the functional response of miRNAtargeted genes to drought stress in apples, we performed a Gene Ontology (GO) enrichment analysis (agriGO, http:// systemsbiology.cau.edu.cn/agriGOv2/) to visualize the enriched biological process, molecular function, and cellular component categories ${ }^{41}$. MiRNA-targeted genes that were differentially expressed between both plant groups under drought were used to draw hierarchical graphs by agriGO.

\section{qRT-PCR validation of miRNAs and their targets}

Stem-loop and regular quantitative reversetranscriptase PCR (qRT-PCR) methods were used to verify the expression of miRNAs and their targets according to MIQE methods ${ }^{42}$, respectively. Briefly, $4 \mu \mathrm{g}$ of total RNA was used to synthesize first-strand cDNA with the RevertAid First Strand cDNA Synthesis Kit
(Thermo Fisher Scientific, MA, USA). The reverse transcription primers for stem-loop qRT-PCR was a mixture of the $M D H$ (malate dehydrogenase) reverse primer and miRNA-specific reverse transcription primer ${ }^{43}$. Oligo-dT primers were used in the regular qRT-PCR. All reactions were performed on the CFX96 Real-Time PCR Detection System (Bio-Rad, CA, USA). Primer efficiency was analyzed by diluting $200 \mathrm{ng}$ cDNA to $40,8,1.6$, and $0.32 \mathrm{ng}$. The three biological replicates were the same as those used for the small RNA-seq in each experiment. The $2^{-\Delta \Delta \mathrm{Ct}}$ method was used to calculate the relative expression of the genes ${ }^{44}$ with $M D H$ as a reference. The data were analyzed using GraphPad Prism 5.0 software (GraphPad Software, CA, USA) and Student's $t$-test was applied to compare two samples. The primers used in this study are listed in Supplementary Table S9.

\section{Gene transformation in apple callus and osmotic stress treatment}

To overexpress miRNAs in apple calli, the miRNA precursor genes (MIR), including sequences located 100-200 bp upstream and downstream of miRNA stem loops, were cloned by PCR from $\mathrm{F}_{1}$ progenies of R3 $(M . \times$ domestica) $\times M$. sieversii and then introduced into the pK2GW7 binary vector by Gateway technology (Invitrogen, CA, USA). The primers used for constructing these vectors are shown in Supplementary Table S9. Wild-type (WT) "Orin" apple calli were transformed by Agrobacterium-mediated transformation (strain EHA105) ${ }^{45}$. Transgenic calli harboring 35S:MIR156p or 35S:MIRn249 were screened on MS agar medium $(1.5 \mathrm{mg} / \mathrm{L} 2,4-$ dichlorophenoxyacetic acid (2,4-D), $0.4 \mathrm{mg} / \mathrm{L}$ 6-BA, $30 \mathrm{~g} /$ $\mathrm{L}$ sucrose, and $8 \mathrm{~g} / \mathrm{L}$ agar) containing $50 \mathrm{mg} / \mathrm{L}$ kanamycin and $250 \mathrm{mg} / \mathrm{L}$ cefotaxime. After serial subcultures, the transgenic or WT calli were used to detect the expression of miRNAs by stem-loop qRT-PCR analysis.

For osmotic stress tolerance, calli with similar sizes were selected and cultured on MS agar medium with or without $150 \mathrm{mM}$ mannitol. After 3 weeks, the callus fresh weight was measured. For target expression in the transgenic calli, MS liquid medium (1.5 mg/L 2,4-D, $0.4 \mathrm{mg} / \mathrm{L} 6-\mathrm{BA}$, and $30 \mathrm{~g} / \mathrm{L}$ sucrose) with or without $150 \mathrm{mM}$ mannitol for $6 \mathrm{~h}$ at $25^{\circ} \mathrm{C}$ in a shaker (120 r.p.m.) was used to extract total RNA.

\section{Results}

\section{Identification of $F_{1}$ plants with different drought stress tolerances}

We identified six $F_{1}$ progenies $(0-293,0-106,0-334$, $7-275,8-134$, and $0-330)$ as drought-tolerant plants and four $(0-297,0-131,1-338$, and 8-11) as droughtsensitive plants (Fig. 1 and Table 1) based on their recovery rate after rehydration. Plants with recovery rates $>50 \%$ were defined as drought-tolerant plants and those 


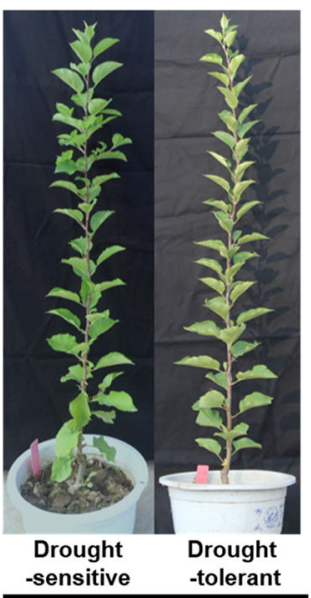

Control

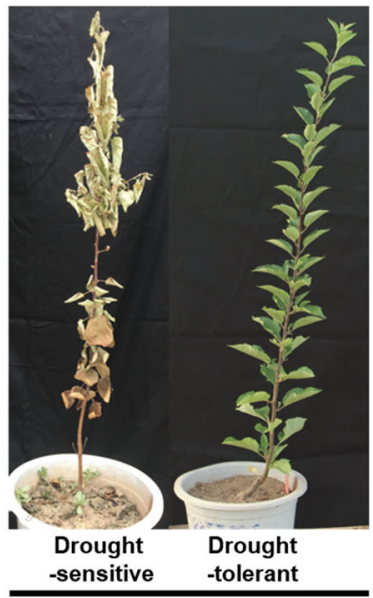

Drought

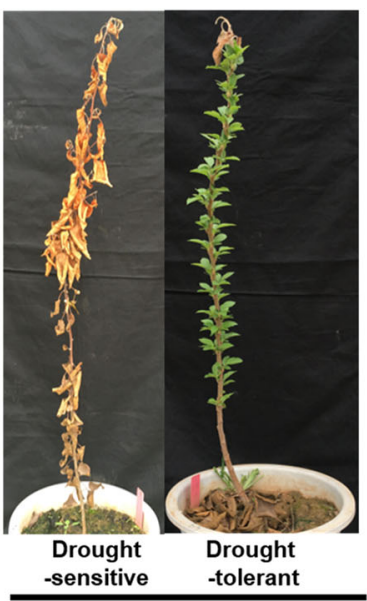

Rehydration

Fig. 1 Phenotypes of drought-sensitive and drought-tolerant plants under control (left), drought for 12 days (middle), or rehydration for 30 days (right). The phenotype of $F_{1}$ progenies of $R 3\left(M\right.$. $\times$ domestica) $\times M$. sieversii is depicted after drought treatment and rehydration. $F_{1}$ progenies with drought-sensitive or drought-tolerant plants were propagated by tissue culture and transplanted to soil for an additional 10 months. The plants were then treated with drought stress for 12 days and rehydrated for 30 days

with recovery rates $<50 \%$ were defined as droughtsensitive plants.

\section{Overview of sRNA sequencing}

Agarose electrophoresis analysis suggested a decent quality of the extracted RNA for each biological replicate of $F_{1}$ progeny under control or drought treatment (Supplementary Fig. S1a). Analysis by agarose electrophoresis and Agilent 2100 also showed a decent quality of the pooled RNA (Supplementary Fig. S1b and c). In addition, the quality of all libraries was good enough for downstream analysis (Supplementary Table S1). We sequenced a total of 368.88 million $(\mathrm{M})$ raw and $356.58 \mathrm{M}$ clean reads, with a mean of $29.71 \mathrm{M}(97 \%)$ clean reads per library (Table 2). For further analysis, we isolated $264.21 \mathrm{M}$ clean reads with lengths ranging from 18 to 30 nt (74\%) and 67.21 M unique reads (25\%) (Supplementary Table S1). All libraries had a similar distribution of lengths $(\sim 72 \%$ for redundant reads and $84 \%$ for unique reads, Fig. 2). The 24 nt reads were the most abundant in redundant reads (34\%) and unique reads (52\%), followed by the $20 \mathrm{nt}$ redundant reads (14\%) and $23 \mathrm{nt}$ unique reads (10\%) (Fig. 2). Among the 18-30 nt sRNA, $83 \%$ redundant reads and $71 \%$ unique reads were successfully mapped to the apple reference genome (Supplementary Table S1).

\section{Identification of known and novel miRNAs in apple}

A total of $4.4 \mathrm{M}$ redundant reads, including 205 precursors and 206 mature miRNAs, were mapped to the miRbase without mismatch (Supplementary Tables S2 and S3).
To identify novel miRNAs in apple plants, a total of $1.3 \mathrm{M}$ redundant reads $(0.57 \%)$ were mapped onto 274 hairpin structures, identifying 253 candidates, among which 123 had miRNA* sequences (Supplementary Tables S2 and S4). As miRNA* is a common tool to verify new miRNAs ${ }^{46}$, we designated the 123 candidates with miRNA* sequences as novel miRNAs and the remaining 130 without miRNA* sequences as novel miRNA candidates. The lengths of the 253 novel apple miRNAs ranged from 18 to $24 \mathrm{nt}$, with a dominant distribution of $24 \mathrm{nt}$ (211 miRNAs), followed by $21 \mathrm{nt}$ (18 miRNAs), $22 \mathrm{nt}$ (12 miRNAs), 23 nt (4 miRNAs), 18 nt (3 miRNAs), 19 nt (3 miRNAs), and 20 nt (2 miRNAs) (Supplementary Table S4).

The identified known and novel miRNAs were classified into 40 families. Among these families, the largest was miR156, with 31 members, followed by miR172 (15 members), miR171_1 (14 members), and miR167_1 (10 members). Only two novel miRNAs were classified into miRNA families: miRn234 into the miR159 family and miRn85 into the miR169_2 family (Fig. 3 and Supplementary Table S5).

In addition to the known and novel miRNA tags, small RNA reads were also mapped to rRNA, tRNA, snRNA, snoRNA, repeat sequences, NAT-siRNA, and genes. The most abundant redundant reads were mapped onto genes (17\%), followed by repeat sequences (16\%), rRNAs ( $8.1 \%)$, and NAT-siRNAs (7.2\%). Unique reads were also most abundant in repeat sequences $(26 \%)$, followed by genes (7.8\%), rRNAs (1.3\%), and NAT-siRNAs (1.2\%). Among unique and redundant reads, the minimum RNA classes were snoRNA $(0.11 \%$ and $0.22 \%$, respectively), snRNA 
Table 2 Overview of miRNA sequencing reads

\begin{tabular}{|c|c|c|c|c|c|c|c|c|}
\hline Sample & & Total reads & $N \%>10 \%$ & Low quality & $\begin{array}{l}\text { 5'-Adapter } \\
\text { contamine }\end{array}$ & $\begin{array}{l}3^{\prime} \text {-Adapter null } \\
\text { or insert null }\end{array}$ & $\begin{array}{l}\text { With poly } A / T / \\
G / C\end{array}$ & Clean reads \\
\hline \multirow{6}{*}{$\begin{array}{l}\text { Drought- } \\
\text { sensitive plants }\end{array}$} & Control 1 & $33,928,632(100 \%)$ & $371(0 \%)$ & $81,721(0.24 \%)$ & $33,287(0.10 \%)$ & $1,377,648(4.1 \%)$ & $53,145(0.16 \%)$ & $32,382,460(95 \%)$ \\
\hline & Control 2 & $31,905,071(100 \%)$ & $360(0 \%)$ & $84,190(0.26 \%)$ & $32,884(0.10 \%)$ & $555,157(1.7 \%)$ & $70,210(0.22 \%)$ & $31,162,270(98 \%)$ \\
\hline & Control 3 & $33,330,848(100 \%)$ & $349(0 \%)$ & $77,489(0.23 \%)$ & $32,009(0.10 \%)$ & $653,356(2.0 \%)$ & $58,660(0.18 \%)$ & $32,508,985(98 \%)$ \\
\hline & Drought 1 & $32,537,917$ (100\%) & $347(0 \%)$ & $82,237(0.25 \%)$ & 27,717 (0.09\%) & $875,074(2.7 \%)$ & $37,609(0.12 \%)$ & $31,514,933(97 \%)$ \\
\hline & Drought 2 & $24,779,273$ (100\%) & $252(0 \%)$ & $66,295(0.27 \%)$ & $25,252(0.10 \%)$ & 709,788 (2.9\%) & $64,004(0.26 \%)$ & $23,913,682(97 \%)$ \\
\hline & Drought 3 & $30,900,174$ (100\%) & $328(0 \%)$ & 75,907 (0.25\%) & $34,188(0.11 \%)$ & $775,123(2.5 \%)$ & $67,303(0.22 \%)$ & $29,947,325(97 \%)$ \\
\hline \multirow{6}{*}{$\begin{array}{l}\text { Drought- } \\
\text { tolerant plants }\end{array}$} & Control 1 & $31,489,922(100 \%)$ & $443(0 \%)$ & $86,581(0.27 \%)$ & $28,714(0.090 \%)$ & $1,352,490(4.3 \%)$ & $39,785(0.13 \%)$ & $29,981,909$ (95\%) \\
\hline & Control 2 & $29,888,571(100 \%)$ & $430(0 \%)$ & $78,512(0.26 \%)$ & 32,389 (0.11\%) & 855,209 (2.9\%) & $58,814(0.20 \%)$ & $28,863,217(97 \%)$ \\
\hline & Control 3 & $26,866,676$ (100\%) & $435(0 \%)$ & $73,980(0.28 \%)$ & 24,177 (0.090\%) & $448,026(1.7 \%)$ & $62,656(0.23 \%)$ & $26,257,402(98 \%)$ \\
\hline & Drought 1 & $29,371,046$ (100\%) & $444(0 \%)$ & $73,889(0.25 \%)$ & $31,098(0.11 \%)$ & $504,062(1.7 \%)$ & $51,860(0.18 \%)$ & $28,709,693(98 \%)$ \\
\hline & Drought 2 & $30,422,600$ (100\%) & $445(0 \%)$ & $85,569(0.28 \%)$ & 26,499 (0.090\%) & $978,522(3.2 \%)$ & $45,285(0.15 \%)$ & $29,286,280(96 \%)$ \\
\hline & Drought 3 & $33,456,352(100 \%)$ & $479(0 \%)$ & $82,842(0.25 \%)$ & $28,253(0.080 \%)$ & $1,266,189(3.8 \%)$ & $29,138(0.090 \%)$ & $32,049,451(96 \%)$ \\
\hline
\end{tabular}

( $0.080 \%$ and $0.10 \%$, respectively), and tRNA (nearly $0 \%$ for both) (Supplementary Table S2).

\section{Expression analyses of the miRNAs in apple}

The miRNAs identified in drought-sensitive or droughttolerant plants in each treatment were classified into six categories of expression based on normalized read counts: no (0 reads), extra-low (0-10 reads), low (10-100 reads), moderate (100-1000 reads), high (1000-10,000 reads), and extra-high (>10,000 reads) expression (Fig. 4). Highly similar percentages of identified miRNAs fell into the same category in each treatment (Fig. 4a). The largest percentage of known miRNAs fell into the low category, followed by the moderate and extra-low categories, whereas the lowest percentages fell into the no expression category (Fig. 4b and Supplementary Table S6). MiR1511 was the most abundant miRNA, with over 60,000 normalized reads in each treatment. The majority of novel miRNAs had low abundances in each treatment (Fig. 4c and Supplementary Table S6). For example, miRn-54 had 0.75 normalized reads in a library of untreated control drought-tolerant plants. Moreover, different members of the same miRNA families displayed different expression levels. For example, miR156h had more than 300 normalized reads, but miR156t had $<10$ (Supplementary Table S6). Notably, 20 miRNAs were absent in at least one treatment, including 12 known and 8 novel miRNAs (Supplementary Table S6). In drought-tolerant plants, miR3627 a/b/c, miRn-50, miR-61, and miRn-158 were not present under drought treatment, whereas miR399 e/f/g/ h, miRn-67, and miRn-203 were not detected under control conditions. In drought-sensitive plants, miR828a and miR828b were absent under both conditions, whereas
miRn-11 and miRn-78 were not present under drought. In addition, miR169c and miR169d were only detected in drought-sensitive plants under drought, whereas miR7128 and miRn-54 were only detected in drought-tolerant plants under control conditions (Supplementary Table S6).

The expression levels of miRNAs in drought-tolerant or drought-sensitive plants under control or drought treatment are provided in Figs. 5 and 6, and Supplementary Table S7. Thirty-nine percent of miRNAs displayed significant expression levels ( $p$-value $<0.05)$ and 93 differentially expressed miRNAs were identified in the drought response. After drought treatment, 21 miRNAs were downregulated and 14 miRNAs were upregulated in drought-sensitive plants, whereas 32 miRNAs were downregulated and 29 miRNAs were upregulated in drought-tolerant plants (Fig. 5a, b). Among these 93 differentially expressed miRNAs, only 3 novel miRNAs (miRn15, miRn85, and miRn246) were responsive to drought in both plant groups (Fig. 6 and Supplementary Table S7). Specifically, miRn15 was upregulated and miRn85 was downregulated in both plants in response to drought, whereas miRn246 was downregulated in drought-tolerant plants but upregulated in droughtsensitive plants in response to drought. Moreover, drought treatment induced most novel miRNAs (76\%) and repressed most known miRNAs (88\%) in droughttolerant plants, but upregulated (71\%) and downregulated (71\%) most known miRNAs in drought-sensitive plants (Fig. 5d and Supplementary Table S7).

When comparing drought-sensitive and droughttolerant plants, 151 miRNAs were differentially expressed under treatment, including 119 miRNAs under 

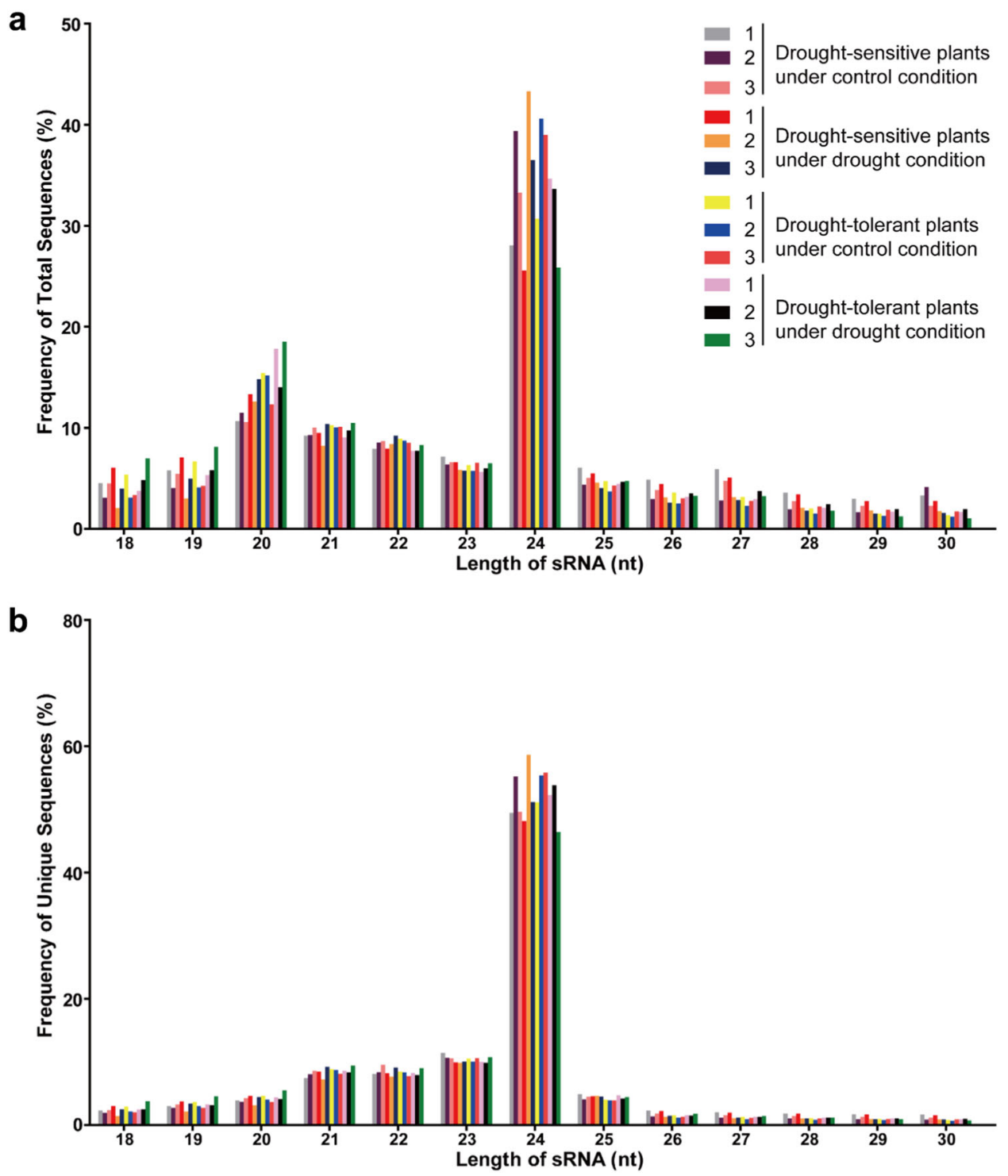

Fig. 2 Mapping status of sRNAs in each library. a redundant sRNAs. $\mathbf{b}$ unique sRNAs. The numbers 1, 2, and 3 indicate three biological replicates

control and 67 miRNAs under drought (Fig. 5a, c). These 67 differentially expressed miRNAs represent a unique response of miRNAs in drought-tolerant plants and may be important miRNAs for improving the drought tolerance of apple rootstocks. Among these 67 miRNAs, 35 were differentially expressed under both normal and drought conditions, including 23 lower abundance and 12 higher abundance miRNAs in drought-tolerant plants (Fig. 5c). Among these miRNAs, miRn-157, miRn-158, and miRn-101 were classified as lower abundance in drought-sensitive plants, whereas miR395 a/b/c/d/e/f/g/ $\mathrm{h} / \mathrm{i}$ were classified as higher abundance (Supplementary Table S7).

In addition, the expression levels of 22 miRNAs that were similar in both plant groups under normal conditions were either reduced or increased after drought treatment, including miR156 aa/p/q/r/s/x/y/z, miR390 a/ b/c/d/e/f, miRn-134, miRn-249, and miR5225 a/b (Supplementary Table S7). Of these 22 miRNAs, nine were responsive to drought in drought-sensitive plants and 13 were responsive to drought in drought-tolerant plants (Figs. 5a and 6).

Under control conditions, 69 miRNAs displayed higher abundance in drought-tolerant plants, whereas $50 \mathrm{miR}$ NAs displayed lower abundance in drought-tolerant plants (Fig. 5c). In drought-tolerant plants relative to drought-sensitive plants, 35 miRNAs were higher in abundance, whereas 32 miRNAs were lower in abundance after drought treatment (Fig. 5c). Under both normal and drought conditions, most known miRNAs were more 


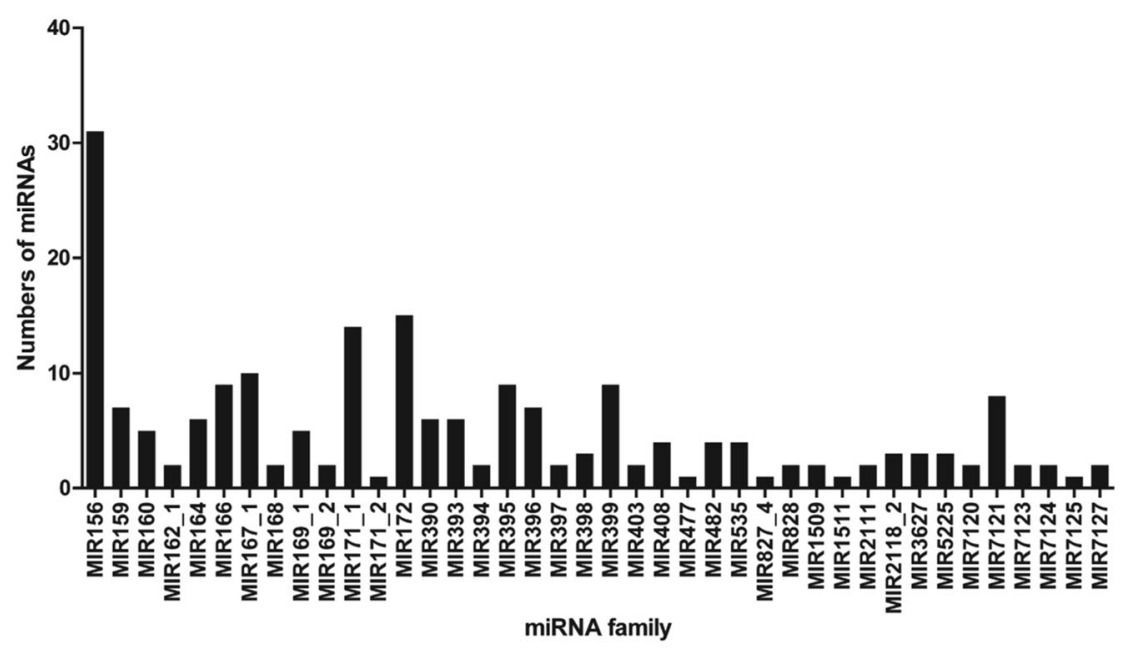

Fig. 3 Number of identified miRNAs in known miRNA families
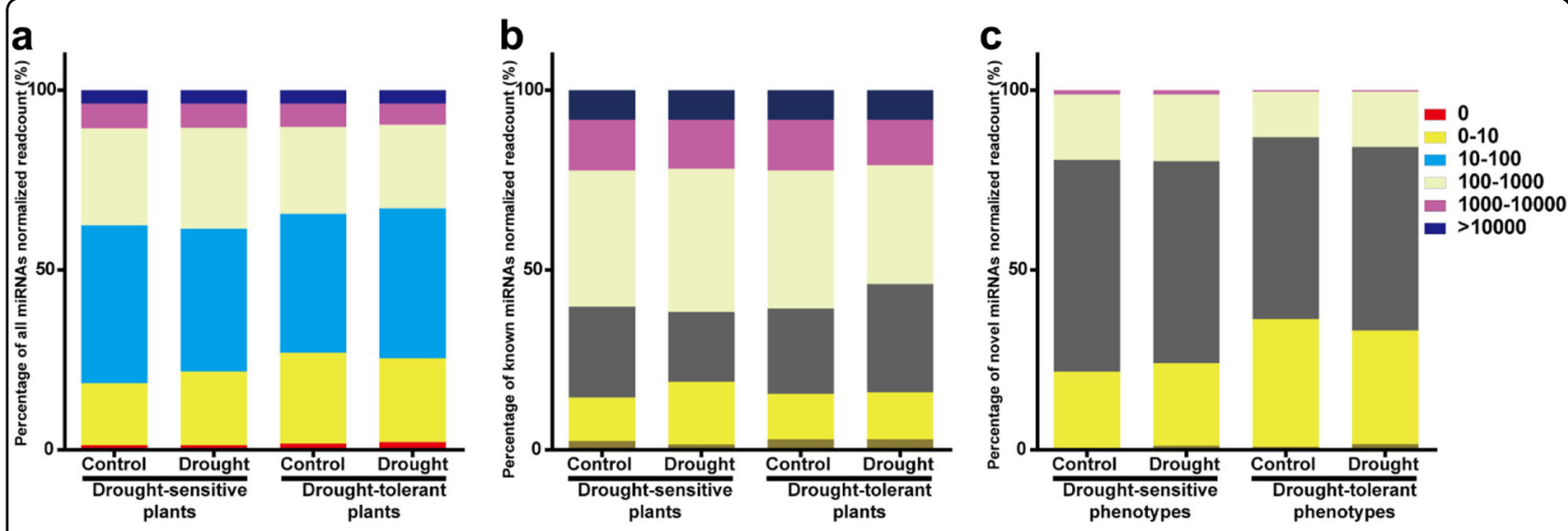

Fig. 4 Expression levels of miRNAs with normalized read count frequencies in each treatment. a all identified (including known and novel) miRNAs. b known miRNAs. c novel miRNAs. Expression level category: 0, no expression; 0-10, extra-low expression; 10-100, low expression; 100-1000, moderate expression; 1000-10,000, high expression; >10,000, extra-high expression

abundant (91\% in control and $74 \%$ in drought treatment), whereas most novel miRNAs were less abundant $(90 \%$ in control and $72 \%$ in drought treatment) in droughttolerant plants than in drought-sensitive plants (Fig. 5e and Supplementary Table S7).

\section{Target prediction and GO analysis}

We predicted 2754 miRNA target pairs, including 1090 unique protein-coding mRNAs. Among the 1090 proteincoding mRNAs, the majority of genes encoded transcription factors (SPL transcription factors, C3HC4-type RING finger), resistance-associated proteins (TIR-NBSLRR class disease resistance protein), transport proteins (sulfate transporter protein), and enzymes, such as kinases (receptor kinase), transferases ( $S$-adenosyl-L-methioninedependent methyltransferases), and phosphatases (RNA polymerase II C-terminal domain phosphatase) (Supplementary Table S8).

GO analysis of 1090 protein-coding mRNAs suggested that enriched biological processes included cellular process (GO:0009987), metabolic process (GO:0008152), and response to stimulus (GO:0050896), whereas binding (GO:0005488) and catalytic activity (GO:0003824) were the most abundant classes in the molecular function category (Fig. 7). The most significant cellular components were cell (GO:0005623), cell part (GO:0044464), and organelle (GO:0043226).

We also evaluated the potential functions of 67 miRNAtargeted genes that were differentially expressed between both plant groups under drought. We selected a total of 1213 miRNA target pairs for GO enrichment analysis, including 390 protein-coding mRNAs. Hierarchical 


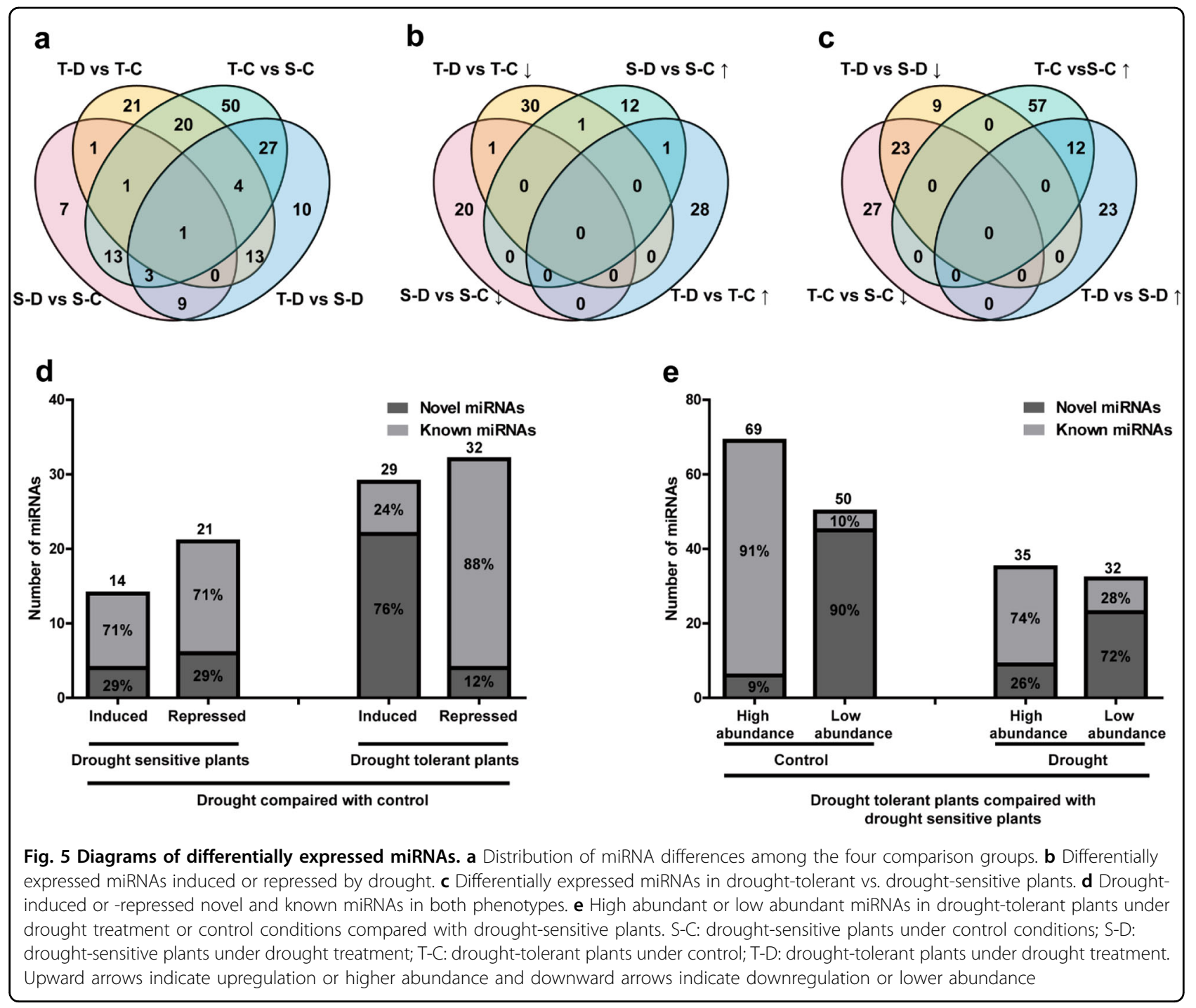

graphs displayed significantly enriched GO terms in biological process and molecular function categories, rather than the cellular component. Within the biological process category, programmed cell death (GO:0012501), defense response (GO:0006952), postembryonic development (GO:0009791), regulation of cellular process (GO:0050794), and biological regulation (GO:0065007) were significantly enriched (Fig. 8). Within the molecular function terms, receptor activity (GO: 0004872), ubiquitin-protein ligase activity (GO: 0004842), transferase activity (GO:0016740), ion binding (GO:0043167), and protein binding (GO: 0005515) were significantly enriched (Fig. 8).

\section{Expression verification of miRNAs and their targets by qRT- PCR}

We used stem-loop qRT-PCR to validate sequencing results by determining miRNA expression levels in both plant groups under control and drought treatments. From the high-throughput sequencing results, we selected eight miRNAs (four conserved and four novel) for stem-loop qRT-PCR, including miR156, miR395, miR408a, miR5225, miRn-101, miRn-157, miRn158, and miRn-249 (Fig. 9). Primer analysis suggested that all stem-loop qRTPCR primers had high efficacy and were thus suitable for miRNA expression detection (Supplementary Fig. S2a and b). The stem-loop qRT-PCR results confirmed the expression of seven of the eight miRNAs (with the exception of miR156), indicating the reliability of our small RNA-seq data. The expression of miR156, miR395, and miR408a was much higher in drought-tolerant plants than in drought-sensitive plants under control or drought conditions. MiR408a expression was induced by drought in both plant groups, whereas miR156 and miR395 were not. Under control conditions, the expression of miR5225 and miRn-249 was similar between drought-tolerant and 


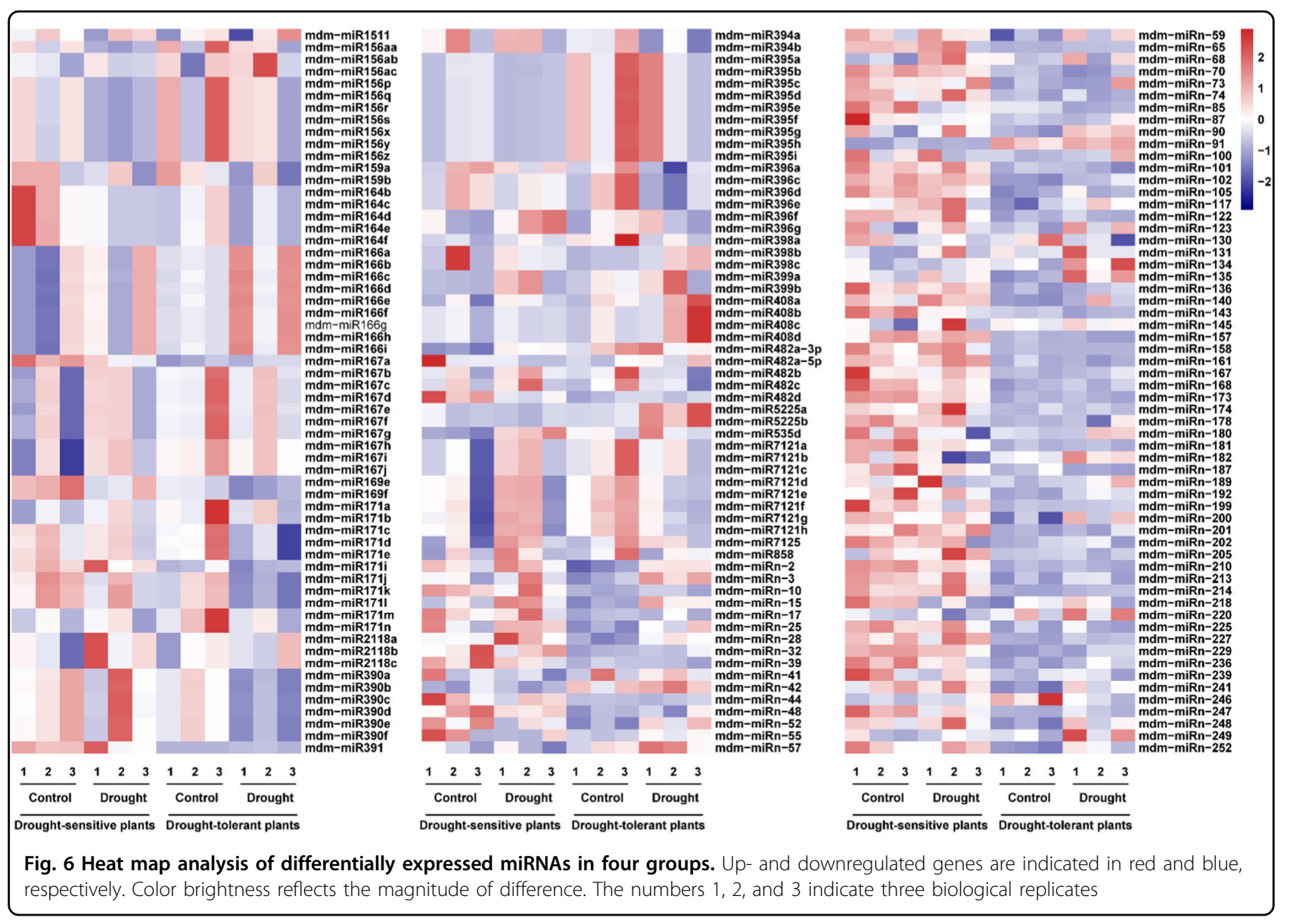

drought-sensitive plants; however, under drought conditions, the increased expression of both miRNAs in drought-tolerant plants was observed. In addition, the expression of both miR5225 and miRn-249 was only induced by drought in drought-tolerant plants. MiRn-101, miRn-157, and miRn-158 had similar expression patterns with lower expression in drought-tolerant plants under both control and drought conditions (Fig. 9). The expression pattern of miR156 by stem-loop qRT-PCR was not consistent with the small RNA-seq results, possibly because stem-loop qRT-PCR detected all miR156 family members (Figs 6 and 9).

We next analyzed the expression patterns of miRNAtargeted genes by using high-efficiency primers (Supplementary Fig. S3a and b), including seven miR156 targets, three miR395 targets, and one novel miRNA (miRn-249) targeted gene. The qRT-PCR results demonstrated that three predicted target genes of miR395 (RNA polymerase II C-terminal domain phosphatase, WRKY33, and receptor kinase) had expression patterns opposite that of miR395 in both plant groups under control and drought conditions. This finding indicated a negative correlation with miR395, supporting the potential of these genes as miR395 targets (Fig. 10). Zinc finger (C3HC4-type RING finger) was predicted as the target of miRn-249 under drought, with an expression pattern that negatively correlated with that of miRn-249 in both plant groups under drought. The expression patterns of SPL genes were more complicated. SPL_4 and SPL_6a displayed expression patterns opposite that of miR156 under control or drought, whereas the expression of $S P L_{-} 5$ and $S P L_{-} 13 a$ was only negatively correlated with that of miR156 transcripts under drought. Thus, both SPL genes may only be targeted by miR156 under drought conditions. SPL_2, $S P L \_9$, and $S P L_{-} 12$ had the same expression pattern as miR156 under control and drought, indicating that these three genes may not be the targets of miR156 in apple rootstocks (Fig. 10).

\section{Function verification of miRNAs in response to osmotic stress}

To verify the function of these miRNAs, we selected two miRNAs, miR156p and miRn-249, and generated transgenic calli carrying 35S:MIR156p or 35S:MIRn-249. As drought stress could cause osmotic stress in plants, we treated the WT and transgenic calli with $150 \mathrm{mM}$ mannitol. Stem-loop qRT-PCR analysis demonstrated that miR156p was upregulated $\sim 400$-fold in the transgenic 


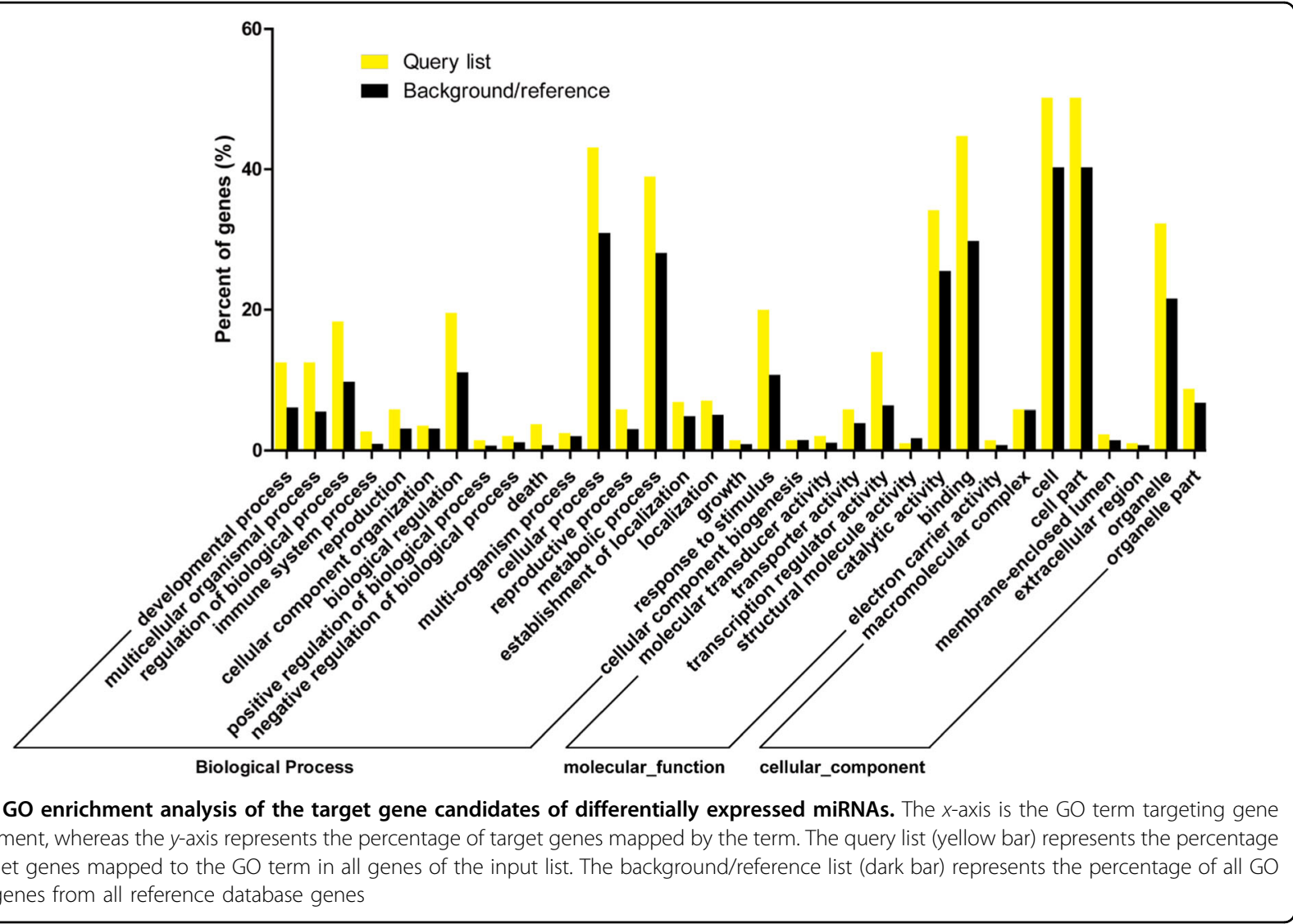

calli compared with that in WT calli and 10-fold for miRn-249 in miRn-249 transgenic calli (Fig. 11a). When treated with $150 \mathrm{mM}$ mannitol for 3 weeks, the relative growth rate of miRn-249 and miR156p transgenic calli was higher than that of WT (Fig. 11b), suggesting positive roles for these two miRNAs in osmotic stress tolerance. We also detected the expression levels of the target genes of these two miRNAs. Our results showed that four target genes of miR156 (SPL_6a, SPL_9, SPL_12, and SPL_13a) were repressed by elevated miR156p expression (Fig. 11c). However, the expression of $S P L \_2$ and Zinc finger (C3HC4-type RING finger), which were predicted as the targets of miR156 and miRn-249, respectively, was not reduced in miR156p or miRn-249 transgenic calli (Supplementary Fig. S4).

\section{Discussion}

Drought is a significant and common environmental stressor, restricting apple yield and quality worldwide. MiRNAs, the negative regulators of gene expression, are associated with abiotic stress ${ }^{47,48}$, and some droughtresponsive miRNAs have been identified in rice ${ }^{10,12}$, Populus $^{13,49}$, Arabidopsis ${ }^{7,8}$, wheat ${ }^{50}$, maize $^{51}$, soybean ${ }^{52}$, peach $^{53}$, and barley ${ }^{54}$. However, there is no study that systematically identifies and describes the expression dynamics of miRNAs in apple trees in response to drought stress using a deep-sequencing approach. In this study, we identified miRNAs important for the drought tolerance of apple rootstocks by evaluating two pools of $F_{1}$ hybrids with contrary drought phenotypes.

During an interspecific cross, gene recombination occurs; thus, $F_{1}$ progenies from a cross of droughttolerant rootstock with drought-sensitive rootstock can exhibit differences in drought resistance. Therefore, some drought-resistant genes can be transferred to certain progeny with other desired phenotypes. These genes may play an important role in improving the drought resistance of apple rootstocks. Due to heterozygosity within the apple genome, the genetic background of $F_{1}$ progenies varies from one to another, even though the $F_{1}$ progenies have the same parents. To avoid any discrepancy in the genetic background between drought-tolerant and drought-sensitive plants, we used pooled RNA from two groups of plants: drought-tolerant plants and droughtsensitive plants. This strategy is commonly applied to normalize the genetic background of heterozygous plants $^{55-57}$. We generated 12 small RNA libraries with three biological replicates to strengthen the power of our small RNA-seq results. Indeed, sRNAs from each library shared a similar distribution pattern (Fig. 2), consistent 


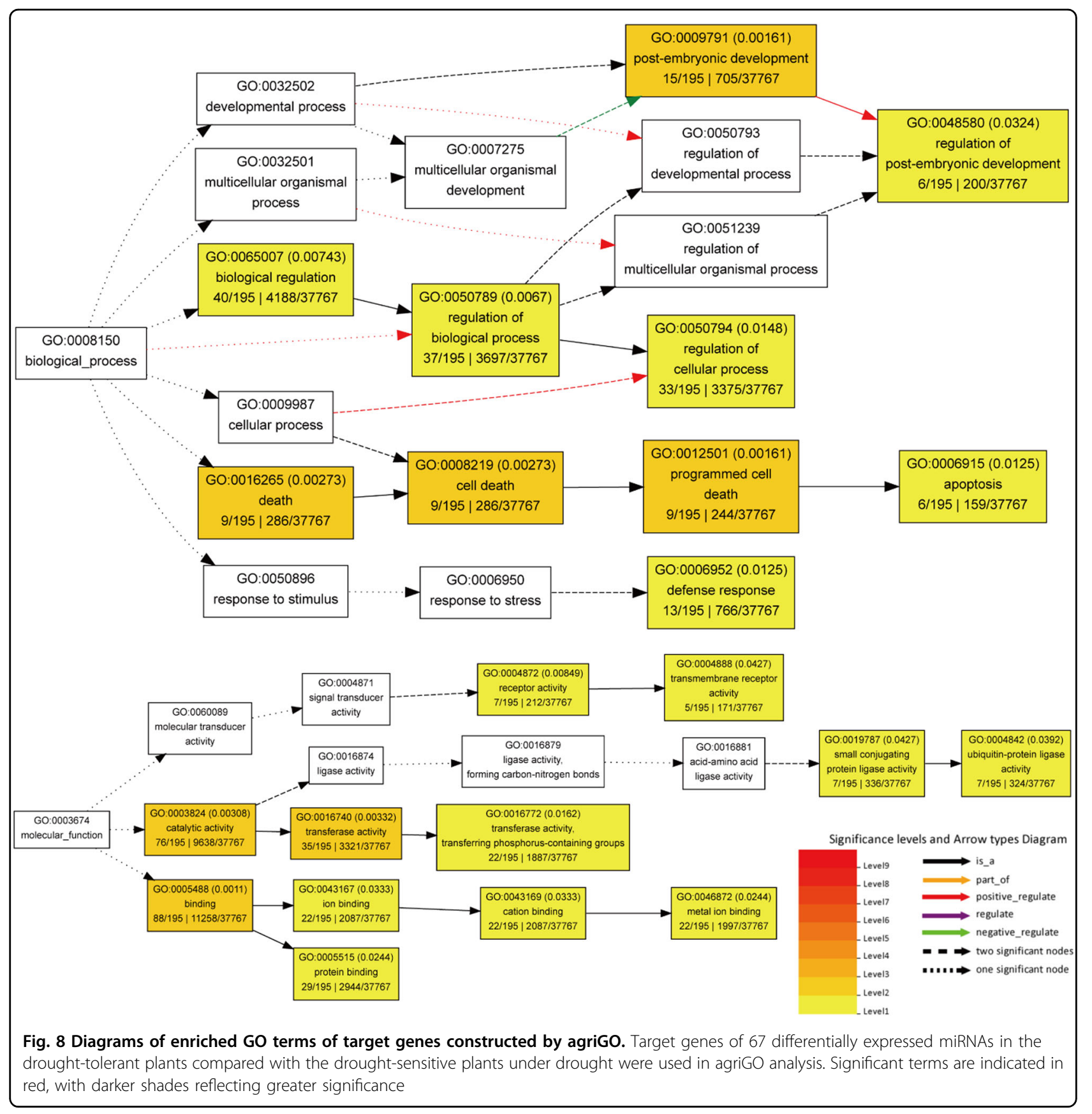

with earlier findings in apple trees ${ }^{58}$. Almost all identified known miRNAs, except miR399c, were matched to the miRbase, suggesting that $F_{1}$ progenies had the same known miRNAs as Golden Delicious, as previously reported $^{58}$. Among these miRNAs, the largest families were consistent with previous research ${ }^{26}$ and the majority of the most abundant miRNAs (such as miR1511, miR166, miR167, and miR396) were conserved across plant species ${ }^{12}$ (Fig. 3).

Many miRNAs are differentially expressed under drought conditions ${ }^{6}$. In our study, 93 miRNAs were determined to be drought-responsive in drought-tolerant and drought-sensitive plants; however, only a few miRNAs had identical response patterns between both plant groups (Fig. 5a, b). This result suggests that most droughtresponsive miRNAs play important, yet different, roles in response to drought in different plants. For example, miR164 was downregulated by drought in droughtsensitive plants but not in drought-tolerant plants. This finding is similar to the downregulation of miR164 observed in M. truncatula ${ }^{59}$ and P. trichocarpa ${ }^{13}$ after drought treatment. Four out of six NAC genes (OMTN2, 


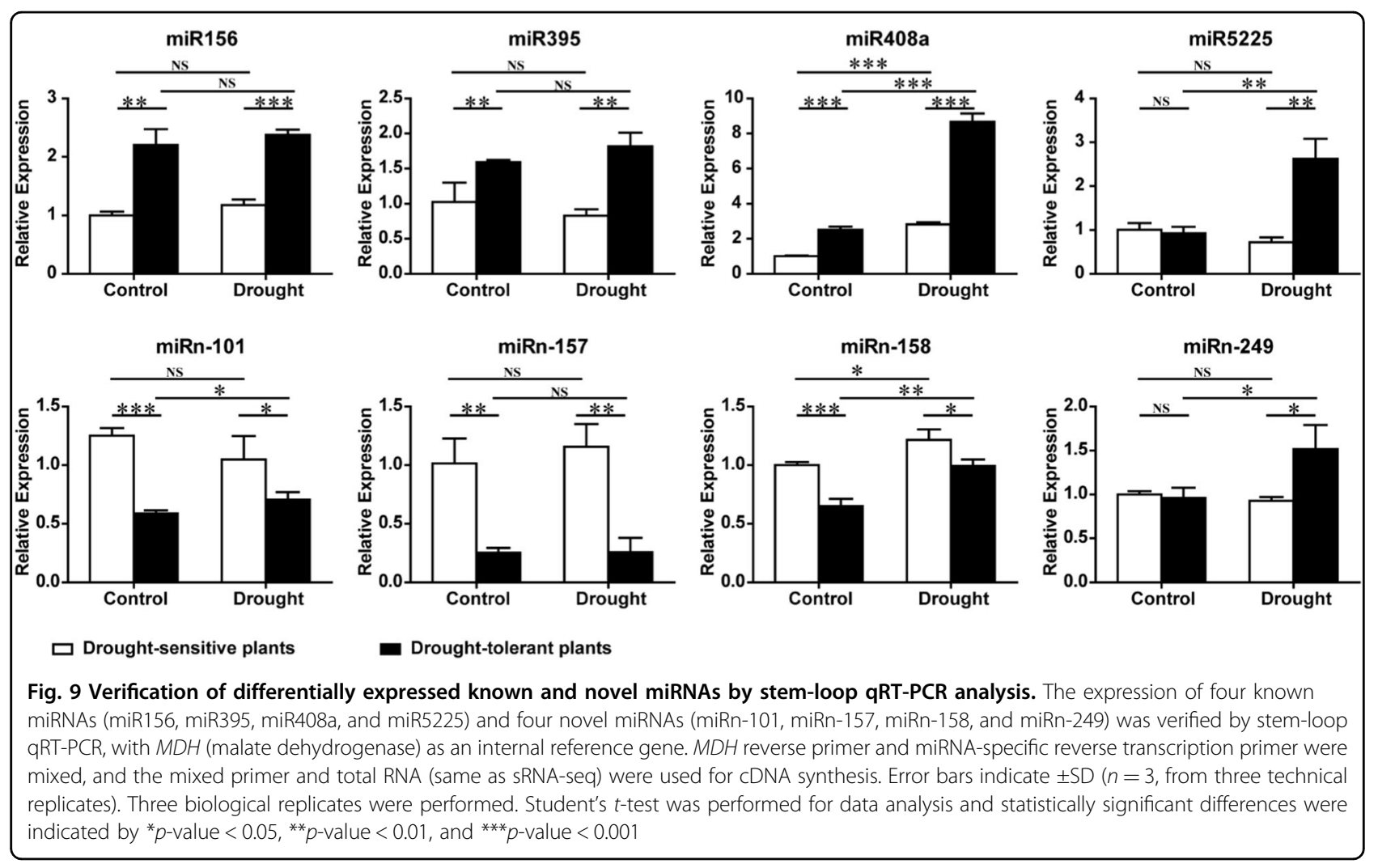

OMTN3, OMTN4, and OMTN6), the targets of miR164, negatively regulate drought resistance ${ }^{60}$, indicating that miR164 may contribute to the drought-sensitive phenotype. However, we did not identify any difference in the expression of miR164 under drought conditions in both plant groups, indicating that miR164 did not contribute to the drought-tolerant phenotype of drought-tolerant plants (Fig. 6 and Supplementary Table S7). In addition, miRn-249 was only induced by drought in droughttolerant plants, with an expression level that was much higher in the same plant group after drought (Fig. 9 and Supplementary Table S7).

The genes associated with C3HC4-type RING finger participate in various plant biological processes ${ }^{61}$, indicating that miRn-249 might play a role in the drought tolerance of Malus. However, there was no difference in the expression levels of Zinc finger and miRn-249 under control conditions between both plant groups, indicating that Zinc finger may only be targeted by miRn-249 under drought conditions. In calli, the increased expression of miRn-249 could improve osmotic stress tolerance (Fig. 11 ), suggesting its positive roles in osmotic stress tolerance. However, the expression of its potential target, Zinc finger (C3HC4-type RING finger), was elevated slightly in transgenic MIRn-249 plants under control or stress conditions (Supplementary Fig. S4b), indicating that this target might not be cleaved by miRn-249 in the callus.
Similarly, SPL_2, one of the targets of miR156p, may not be cleaved by miR156 in the calli. Zinc finger (C3HC4-type RING finger) may have been cleaved by miRn-249, as demonstrated by its lower expression in drought-tolerant plants after exposure to drought for 6 days (Fig. 10). We thus hypothesized that there might be other targets of miRn-249 in the calli, and that the positive role of miRn249 in osmotic stress in calli was not via Zinc finger (C3HC4-type RING finger).

Some miRNAs that were not drought-responsive may still contribute to the drought tolerance of apple rootstock. The expression level of some novel miRNAs (miRn157, miRn-158, and miRn-101) was lower in droughttolerant than drought-sensitive plants under control or drought conditions (Fig. 9). The predicted target of miRn157 is a member of the AP2/ERF and B3 domaincontaining transcription factor family, which are involved in abiotic stress tolerance ${ }^{62,63}$. For example, the overexpression of TSRF1, an ERF transcription factor, improves the drought tolerance of rice ${ }^{64}$; thus, miRn-157 might contribute to the phenotype of drought-tolerant plants in response to drought. The functions of the predicted targets of both miRn-158 and miRn-101 are unknown, but the difference in the expression of both miRNAs between drought-sensitive and drought-tolerant plants under drought indicates that both molecules may participate in the drought response of apple rootstock. 


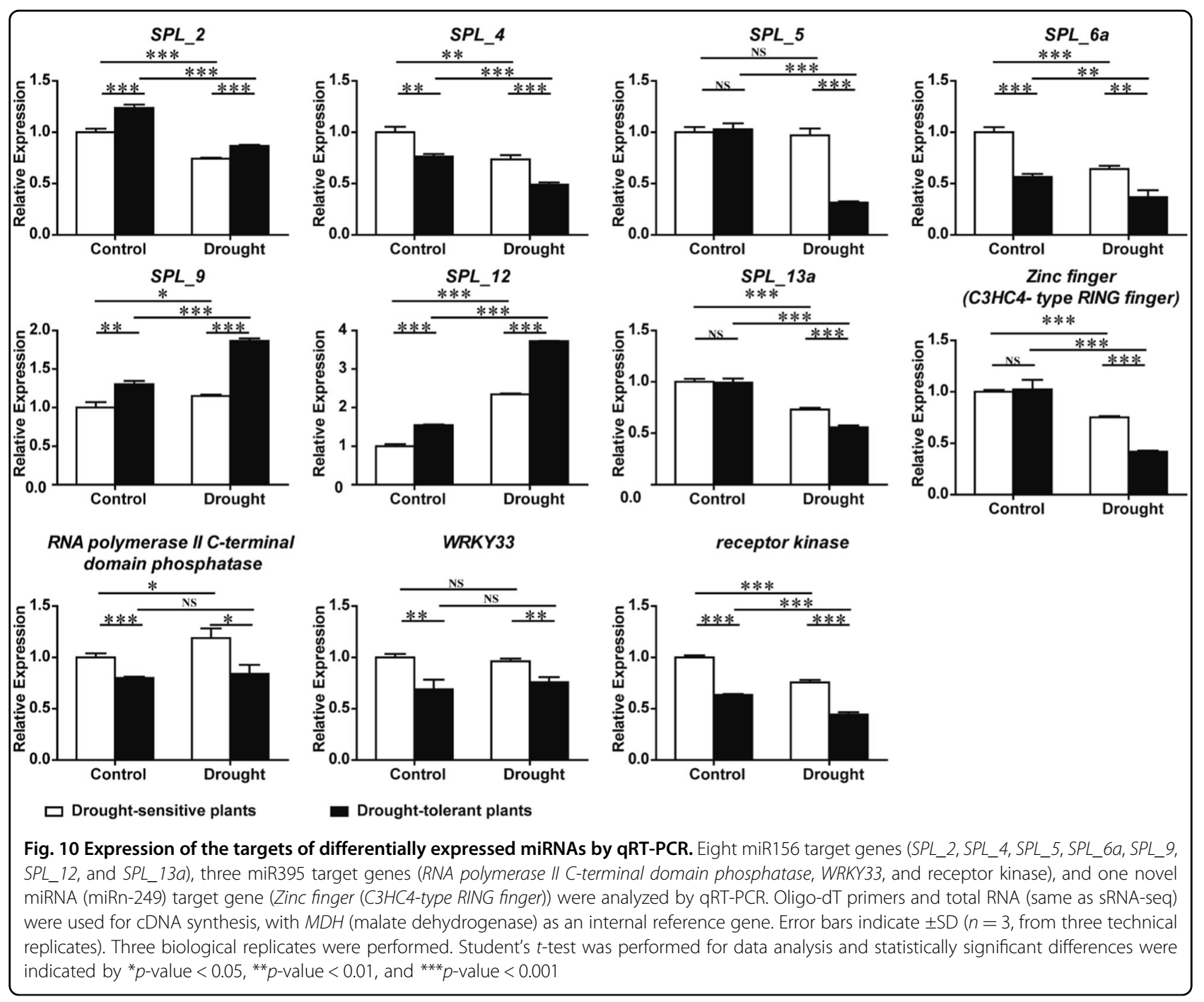

Alternatively, there may be an unknown mechanism for the improvement of drought tolerance in apple rootstock.

The stem-loop qRT-PCR results showed that miR156 expression in drought-tolerant plants was higher than that in drought-sensitive plants; however, miR156 was not induced by drought in either plant group (Fig. 9). This result diverges from those observed under drought conditions in other plant species, such as wheat ${ }^{65}$, where miR156 expression was induced. Indeed, our small RNAseq results suggest that miR $156 \mathrm{p} / \mathrm{q} / \mathrm{r} / \mathrm{s}$ transcripts were reduced under drought in drought-sensitive plants but remained stable in drought-tolerant plants (Fig. 6 and Supplementary Table S7). This phenomenon might be a result of several miR156 tags being detected simultaneously in the stem-loop qRT-PCR analysis, which might potentially affect each other.

Identifying differentially expressed miRNAs in both plant groups under drought conditions is critical to understand the genetic mechanisms underlying the drought tolerance of apple rootstocks. The GO enrichment analysis of predicted targets of differentially expressed miRNAs demonstrated that the enriched biological processes were mainly stress-related (Fig. 8). In wheat leaves, compared with the drought-sensitive genotype, drought-induced programmed cell death in drought-tolerant genotypes promoted higher levels of peroxidase, superoxide dismutase, catalase activities, and ascorbate content under drought stress ${ }^{66}$. Plant development can also contribute to drought tolerance. In sorghum, drought adaptation is associated with canopy development and root growth ${ }^{67}$. In molecular function, ubiquitin-protein ligase activity is associated with drought in Arabidopsis, where the expression of RZFP34/CHYR1, a ubiquitin E3 ligase, is significantly induced by drought. Loss-of-function and gain-of-function analyses of RZFP34/ CHYR1 have suggested that RZFP34/CHYR1 promotes drought tolerance ${ }^{68}$.

Target prediction and qRT-PCR determined that RNA polymerase II C-terminal domain phosphatase, WRKY33, 


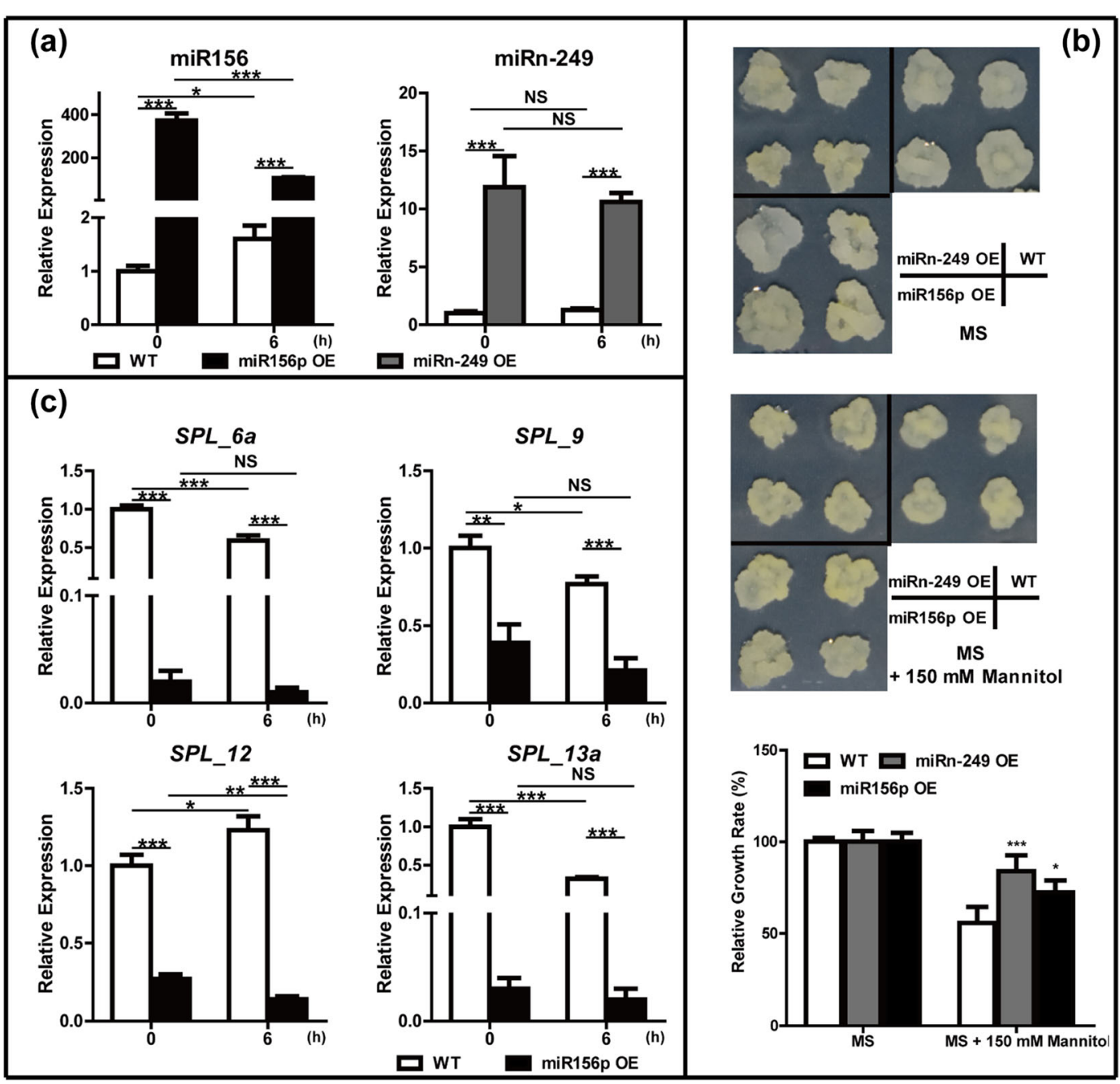

Fig. 11 Effect of osmotic stress in callus derived from WT, miR156p-, and miRn-249-overexpressing callus. a Expression levels of miR156p or miRn-249 in overexpressing calli under control and $150 \mathrm{mM}$ mannitol treatment for $6 \mathrm{~h}$. b Morphology of the calli after culture for 3 weeks on MS medium (upper) or MS medium supplemented with $150 \mathrm{mM}$ mannitol (middle), and relative growth rate between them (under, error bars indicate \pm SD, $n \geq 10$ ). $\mathbf{c}$ Expression levels of the miR156p target genes under control and $150 \mathrm{mM}$ mannitol treatment for $6 \mathrm{~h}$. Stem-loop qRT-PCR and qRTPCR used MDH (malate dehydrogenase) as an internal reference gene, error bars indicate $\pm S D(n=3$, from three technical replicates) and three biological replicates were performed. Student's $t$-test was performed for data, and statistically significant differences were indicated by ${ }^{*} p$-value $<$ $0.05,{ }^{* *} p$-value $<0.01$, and ${ }^{* * *} p$-value $<0.001$

and receptor kinase are likely cleaved by miR395 (Figs. 9 and 10). WRKY33 protein plays an essential role in various biotic and abiotic stresses ${ }^{69,70}$. The homologous gene of receptor kinase in Arabidopsis is a leucine-rich repeat protein (LRR-RLK family member, AT4G23740), whose function in response to drought is currently unknown, but this protein may play a critical role in plant drought tolerance, consistent with other LRR-RLK family members. The overexpression of OsSIK1, an RLK gene, improved drought tolerance in rice. This finding is further supported by knockout transgenic plants showing the drought-sensitive phenotype ${ }^{71}$. Transcripts of miR395 were more abundant in drought-tolerant than drought- sensitive plants under control and drought conditions (Fig. 9). Thus, the downregulation of its target genes may contribute to drought tolerance.

SPL transcription factors are known miR156 targets across plant species ${ }^{58,72,73}$ and these molecules play central roles in plant growth and development ${ }^{21}$. We found the expression pattern of $S P L_{-} 4$ and $S P L_{-} 6 a$ is opposite that of miR156, suggesting that miR156 negatively regulated SPL_4 and SPL_6a under both control and drought conditions. SPL_5 and SPL_13a were only downregulated in drought-tolerant plants after drought, suggesting that, under drought stress, these three SPLs may be important for a miR156-mediated drought response (Fig. 10). In 
Arabidopsis, SPL3, SPL4, and SPL5 (SPL 3/4/5) potentiate the FLOWERING LOCUS T (FT)-FD module in photoperiodic flowering ${ }^{74}$. Early flowering is an adaptive strategy for plants to escape drought ${ }^{20}$; hence, the differential expression of miR156 in both plant groups of Malus under drought indicated that miR156 may participate in the drought stress response through $S P L_{-} 4$ and $S P L_{-} 5$ in apple rootstocks. In tobacco, SPL_6 is critical for N TIRNB-LRR receptor-mediated plant innate immunity ${ }^{75}$. Furthermore, the alleviation of drought tolerance can be pathogen-mediated. For example, infection with the Cucumber mosaic virus improves drought tolerance in Capsicum annum, Solanum lycopersicum, and Nicotiana tabacum $^{76}$, indicating that the upregulation of miR156 may improve the drought tolerance of drought-tolerant plants by targeting SPL6 in apple. In alfalfa, miR156 promotes drought tolerance by silencing $S P L_{-} 13^{77}$. The downregulation of SPL_13a in drought-tolerant plants under drought suggests that miR156 may improve the drought tolerance of apple rootstock by silencing $S P L_{-} 13 a$ (Fig. 10). In addition, when MIR156p was overexpressed in apple calli, the expression levels of $S P L_{-} 6 a, S P L_{-}$, $S P L_{-} 12$, and $S P L_{-} 13 a$ but not $S P L_{-} 2$, were significantly reduced under control or osmotic stress conditions (Fig. 11 and Supplementary Fig. S4), indicating that SPL_2 might not be a target of miR156p in calli.

In summary, our study provides a foundation for further exploration of the candidate miRNAs and their target mRNAs associated with drought response. Although we verified the roles of miR156 and miRn-249 in osmotic stress tolerance in calli, we will characterize the roles of more miRNAs with contrasting expression patterns in both genotypes in response to drought. We will use multiple approaches to explore the molecular mechanisms of these miRNAs in response to drought in apple. Future work should experimentally demonstrate the interactions between miRNAs and their target genes and the mechanisms underlying their roles in apple rootstock drought response, which will be useful for breeding drought-resistant dwarfing rootstocks.

\section{Conclusions}

We examined the drought tolerance of $\mathrm{F}_{1}$ progenies of $\mathrm{R} 3 \times M$. sieversii and the expression patterns of miRNAs in response to drought in drought-tolerant and droughtsensitive plants. Relative to drought-sensitive plants, 67 miRNAs were differentially expressed in drought-tolerant plants under drought conditions. Under drought stress, 61 and 35 miRNAs were differentially expressed in droughttolerant and drought-sensitive plants, respectively. Go analysis demonstrated that predicted target genes were predominately associated with response to stimulus, cellular process, and metabolic process. This work provides a foundation for further developing a comprehensive understanding of the molecular networks involving miRNAs and their targets in response to drought stress in apple.

\section{Acknowledgements}

We thank Dr Yujin Hao and Dr Chunxiang You from Shandong Agricultural University for providing wild-type "Orin" apple calli. This work was supported by the National Natural Science Foundation of China (31622049), the Project of Shaanxi Provincial Youth Science and Technology Star Plan (2015kjxx-14), and the Key Program of the National Natural Science Foundation of China (31330068). We thank the High Performance Computing platform of Northwest A\&F University.

\section{Author contributions}

Q.G., C.N., and H.L. designed the project. C.N., H.L., L.J., D.G., Y.X., Y.Y., X.S., and P. C. performed the experiments. C.N., H.L., Y.X., P.C., and F.M. analyzed the data. C.L. and J.D. provided the hybrid materials. C.N. and H.L. wrote the manuscript.

\section{Conflict of interest}

The authors declare that they have no conflict of interest.

Supplementary Information accompanies this paper at (https://doi.org/ 10.1038/s41438-019-0157-z).

Received: 29 December 2017 Revised: 28 February 2019 Accepted: 5 April 2019

Published online: 08 June 2019

\section{References}

1. Gu, S. \& Kay, M. A. How do miRNAs mediate translational repression? Silence $\mathbf{1}$, 11 (2010).

2. Zamore, P. D. \& Haley, B. Ribo-gnome: the big world of small RNAs. Science 309, 1519-1524 (2005).

3. Bonnet, E., Van de Peer, Y. \& Rouzé, P. The small RNA world of plants. New Phytol. 171, 451-468 (2006).

4. Zhu, J. K. Reconstituting plant miRNA biogenesis. Proc. Natl Acad. Sci. USA 105, 9851-9852 (2008).

5. Carrington, J. C. \& Ambros, V. Role of microRNAs in plant and animal development. Science 301, 336 (2003).

6. Sunkar, R., Li, Y. F. \& Jagadeeswaran, G. Functions of microRNAs in plant stress responses. Trends Plant Sci. 17, 196-203 (2012).

7. Sunkar, R. \& Zhu, J.-K. Novel and stress-regulated microRNAs and other small RNAs from Arabidopsis. Plant Cell 16, 2001-2019 (2004).

8. Liu, H. H., Tian, X. Li, Y. J., Wu, C. A. \& Zheng, C. C. Microarray-based analysis of stress-regulated microRNAs in Arabidopsis thaliana. RNA 14, 836-843 (2008).

9. Li, W.-X. et al. The Arabidopsis NFYA5 transcription factor is regulated transcriptionally and posttranscriptionally to promote drought resistance. Plant Cell 20, 2238-2251 (2008)

10. Zhou, L. et al. Genome-wide identification and analysis of drought-responsive microRNAs in Oryza sativa. J. Exp. Bot. 61, 4157-4168 (2010).

11. Zhao, B. et al. Identification of drought-induced microRNAs in rice. Biochem. Biophys. Res. Commun. 354, 585-590 (2007).

12. Cheah, B. H., Nadarajah, K, Divate, M. D. \& Wickneswari, R. Identification of four functionally important microRNA families with contrasting differential expression profiles between drought-tolerant and susceptible rice leaf at vegetative stage. BMC Genom. 16, 692 (2015).

13. Shuai, P., Liang, D., Zhang, Z., Yin, W. \& Xia, X. Identification of droughtresponsive and novel Populus trichocarpa microRNAs by high-throughput sequencing and their targets using degradome analysis. BMC Genom. 14, 233 (2013).

14. Lu, S., Sun, Y. H. \& Chiang, V. L. Stress-responsive microRNAs in Populus. Plant J. 55, 131-151 (2008).

15. Candar-Cakir, B., Arican, E. \& Zhang, B. Small RNA and degradome deep sequencing reveals drought-and tissue-specific microRNAs and their important roles in drought-sensitive and drought-tolerant tomato genotypes. Plant Biotechnol. J. 14, 1727-1746 (2016).

16. Xie, F., Wang, Q., Sun, R. \& Zhang, B. Deep sequencing reveals important roles of microRNAs in response to drought and salinity stress in cotton. J. Exp. Bot. 66, 789-804 (2015). 
17. Ma, C., Burd, S. \& Lers, A. miR408 is involved in abiotic stress responses in Arabidopsis. Plant J. 84, 169-187 (2015).

18. Trindade, I., Capitão, C., Dalmay, T., Fevereiro, M. P. \& Santos, D. Md miR398 and miR408 are up-regulated in response to water deficit in Medicago truncatula. Planta 231, 705-716 (2010).

19. Bohnert, H. J., Nelson, D. E. \& Jensen, R. G. Adaptations to environmental stresses. Plant Cell 7, 1099-1111 (1995).

20. Han, Y., Zhang, X., Wang, Y. \& Ming, F. The suppression of WRKY44 by GIGANTEA-miR172 pathway is involved in drought response of Arabidopsis thaliana. PLOS ONE 8, e73541 (2013).

21. Chen, $X$. et al. SQUAMOSA promoter-binding protein-like transcription factors: star players for plant growth and development. J. Integr. Plant Biol. 52 946-951 (2010)

22. Wang, J.-W., Czech, B. \& Weigel, D. miR156-regulated SPL transcription factors define an endogenous flowering pathway in Arabidopsis thaliana. Cell $\mathbf{1 3 8}$ 738-749 (2009).

23. Ding, Y., Tao, Y. \& Zhu, C. Emerging roles of microRNAs in the mediation of drought stress response in plants. J. Exp. Bot. 64, 3077-3086 (2013).

24. Li, C. et al. Silencing the SPMPK1, SPMPK2, and SPMPK3 genes in tomato reduces abscisic acid-mediated drought tolerance. Int. J. Mol. Sci. 14, 21983-21996 (2013)

25. Naor, A., Naschitz, S., Peres, M. \& Gal, Y. Responses of apple fruit size to tree water status and crop load. Tree Physiol. 28, 1255-1261 (2008).

26. Xing, L. et al. Shoot bending promotes flower bud formation by miRNAmediated regulation in apple (Malus domestica Borkh.). Plant Biotechnol. J. 14, 749-770 (2016)

27. Guo, X. et al. Small RNA-sequencing links physiological changes and RdDM process to vegetative-to-floral transition in apple. Front. Plant Sci. 8, 873 (2017)

28. Jia-Long, Y. et al. A microRNA allele that emerged prior to apple domestication may underlie fruit size evolution. Plant J. 84, 417-427 (2015).

29. Ma, C et al. Cloning and characterization of miRNAs and their targets, including a novel miRNA-targeted NBS-LRR protein class gene in apple (Golden Delicious). Mol. Plant 7, 218-230 (2014).

30. Kaja, E. et al. Identification of apple miRNAs and their potential role in fire blight resistance. Tree Genet. Genomes 11, 812 (2014).

31. Feng, H. et al. microRNAs and their targets in apple (Malus domestica cv. "Fuji") involved in response to infection of pathogen Valsa mali. Front. Plant Sci. 8 2081 (2017).

32. Liu, B. et al. Influence of rootstock on antioxidant system in leaves and roots of young apple trees in response to drought stress. Plant Growth Regul. 67, 247-256 (2012).

33. Abe, K., Kotoda, N., Kato, H. \& Soejima, J.-i Genetic studies on resistance to Valsa canker in apple: genetic variance and breeding values estimated from intraand inter-specific hybrid progeny populations. Tree Genet. Genomes 7, 363-372 (2011).

34. Langmead, B. \& Salzberg, S. L. Fast gapped-read alignment with Bowtie 2. Nat Methods 9, 357-359 (2012)

35. Li, X. et al. Characterization and comparative profiling of the small RNA transcriptomes in two phases of flowering in Cymbidium ensifolium. BMC Genom. 16, 622 (2015).

36. Friedländer, M. R., Mackowiak, S. D., Li, N., Chen, W. \& Rajewsky, N. miRDeep2 accurately identifies known and hundreds of novel microRNA genes in seven animal clades. Nucleic Acids Res. 40, 37-52 (2012).

37. Moxon, S. et al. A toolkit for analysing large-scale plant small RNA datasets. Bioinformatics 24, 2252-3 (2008).

38. Wen, M., Shen, Y., Shi, S. \& Tang, T. miREvo: an integrative microRNA evolutionary analysis platform for next-generation sequencing experiments. BMC Bioinformatics 13, 140 (2012).

39. Zhou, L. et al. Integrated profiling of microRNAs and mRNAs: microRNAs located on Xq27.3 associate with clear cell renal cell carcinoma. PLoS ONE 5, e15224 (2011).

40. Dai, X. \& Zhao, P. X. psRNATarget: a plant small RNA target analysis server. Nucleic Acids Res. 39, W155-W159 (2011).

41. Tian, T. et al. agriGOv2.0: a GO analysis toolkit for the agricultural community, 2017 update. Nucleic Acids Res. 45, W122-W129 https://doi.org/10.1093/nar/ gkx382 (2017)

42. Bustin, S. A. Why the need for qPCR publication guidelines?-The case for MIQE. Methods 50, 217-226 (2010).

43. Varkonyi-Gasic, E., Wu, R., Wood, M., Walton, E. F. \& Hellens, R. P. Protocol: a highly sensitive RT-PCR method for detection and quantification of microRNAs. Plant Methods 3, 12 (2007).
44. Livak, K. J. \& Schmittgen, T. D. Analysis of relative gene expression data using real-time quantitative PCR and the 2(-Delta Delta C(T)) method. Methods $\mathbf{2 5}$, 402-408 (2001).

45. Wang, $H$. et al. Comprehensive genomic analysis of the TYROSINE AMINOTRANSFERASE (TAT) genes in apple (Malus domestica) allows the identification of MdTAT2 conferring tolerance to drought and osmotic stresses in plants. Plant Physiol. Biochem. 133, 81-91 (2018).

46. Meyers, B. C. et al. Criteria for annotation of plant MicroRNAs. Plant Cell 20 3186-3190 (2008).

47. Guan, Q., Lu, X., Zeng, H., Zhang, Y. \& Zhu, J. Heat stress induction of miR398 triggers a regulatory loop that is critical for thermotolerance in Arabidopsis. Plant J. 74, 840-851 (2013).

48. Jones-Rhoades, M. W. \& Bartel, D. P. Computational identification of plant microRNAs and their targets, including a stress-induced miRNA. Mol. Cell 14 787-799 (2004)

49. Li, B., Qin, Y., Duan, H., Yin, W. \& Xia, X. Genome-wide characterization of new and drought stress responsive microRNAs in Populus euphratica. J. Exp. Bot. 62, 3765-3779 (2011)

50. Yao, Y. et al. Non-coding small RNAs responsive to abiotic stress in wheat (Triticum aestivum L.). Funct. Integr. Genom. 10, 187-190 (2010).

51. Zhang, L. et al. A genome-wide characterization of microRNA genes in maize PLoS Genet. 5, e1000716 (2009).

52. Kulcheski, F. R. et al. Identification of novel soybean microRNAs involved in abiotic and biotic stresses. BMC Genom. 12, 307 (2011).

53. Eldem, V. et al. Genome-wide identification of miRNAs responsive to drought in peach (Prunus persica) by high-throughput deep sequencing. PLOS ONE 7, e50298 (2012).

54. Hackenberg, M., Gustafson, P., Langridge, P. \& Shi, B. J. Differential expression of microRNAs and other small RNAs in barley between water and drought conditions. Plant Biotechnol. J. 13, 2-13 (2015).

55. Yates, S. A. et al. De novo assembly of red clover transcriptome based on RNASeq data provides insight into drought response, gene discovery and marker identification. BMC Genom. 15, 453 (2014).

56. Bai, Y., Dougherty, L., Cheng, L., Zhong, G.-Y. \& Xu, K. Uncovering co-expression gene network modules regulating fruit acidity in diverse apples. BMC Genom. 16, 612 (2015).

57. Wang, N. et al. Comparative transcriptomes analysis of red- and white-fleshed apples in an F1 population of Malus sieversii f. niedzwetzkyana crossed with $M$. domestica 'Fuji'. PLOS ONE 10, e0133468 (2015).

58. Xia, R., Zhu, H., An, Y.-q, Beers, E. P. \& Liu, Z. Apple miRNAs and tasiRNAs with novel regulatory networks. Genome Biol. 13, R47 (2012).

59. Wang, T., Chen, L., Zhao, M., Tian, Q. \& Zhang, W.-H. Identification of droughtresponsive microRNAs in Medicago truncatula by genome-wide highthroughput sequencing. BMC Genom. 12, 367 (2011).

60. Fang, Y., Xie, K. \& Xiong, L. Conserved miR164-targeted NAC genes negatively regulate drought resistance in rice. J. Exp. Bot. 65, 2119-2135 (2014).

61. Jung, Y. et al. BrRZFP1 a Brassica rapa C3HC4-type RING zinc finger protein involved in cold, salt and dehydration stress. Plant Biol. 15, 274-283 (2013).

62. Abogadallah, G. M., Nada, R. M., Malinowski, R. \& Quick, P. Overexpression of HARDY, an AP2/ERF gene from Arabidopsis, improves drought and salt tolerance by reducing transpiration and sodium uptake in transgenic Trifolium alexandrinum L. Planta 233, 1265-1276 (2011).

63. Licausi, F., Ohme-Takagi, M. \& Perata, P. APETALA2/ethylene responsive factor (AP2/ERF) transcription factors: mediators of stress responses and developmental programs. New Phytol. 199, 639-649 (2013).

64. Quan, R. et al. Overexpression of an ERF transcription factor TSRF1 improves rice drought tolerance. Plant Biotechnol. J. 8, 476-488 (2010).

65. Bakhshi, B. et al. The contrasting microRNA content of a drought tolerant and a drought susceptible wheat cultivar. J. Plant Physiol. 216, 35-43 (2017).

66. Hameed, A., Goher, M. \& labal, N. Drought induced programmed cell death and associated changes in antioxidants, proteases, and lipid peroxidation in wheat leaves. Biol. Plant 57, 370-374 (2013).

67. Borrell, A. K. et al. Drought adaptation of stay-green sorghum is associated with canopy development, leaf anatomy, root growth, and water uptake. J. Exp. Bot. 65, 6251-6263 (2014).

68. Ding, S., Zhang, B. \& Qin, F. Arabidopsis RZFP34/CHYR1, a ubiquitin E3 ligase, regulates stomatal movement and drought tolerance via SnRK2. 6-mediated phosphorylation. Plant Cell 27, 3228-3244 (2015).

69. Golldack, D., Lüking, I. \& Yang, O. Plant tolerance to drought and salinity: stress regulating transcription factors and their functional significance in the cellular transcriptional network. Plant Cell Rep. 30, 1383-1391 (2011). 
70. Li, S., Fu, Q., Chen, L., Huang, W. \& Yu, D. Arabidopsis thaliana WRKY25, WRKY26, and WRKY33 coordinate induction of plant thermotolerance. Planta $\mathbf{2 3 3}$ 1237-1252 (2011).

71. Ouyang, S. Q. et al. Receptor-like kinase OsSIK1 improves drought and salt stress tolerance in rice (Oryza sativa) plants. Plant J. 62, 316-329 (2010).

72. Xie, K., Wu, C. \& Xiong, L. Genomic organization, differential expression, and interaction of SQUAMOSA promoter-binding-like transcription factors and microRNA156 in rice. Plant Physiol. 142, 280-293 (2006).

73. Song, $C$. et al. miRNA and degradome sequencing reveal miRNA and their target genes that may mediate shoot growth in spur type mutant "Yanfu 6". Front. Plant Sci. 8, 441 (2017)
74. Jung, J.-H., Lee, H.-J., Ryu, J. Y. \& Park, C.-M. SPL3/4/5 integrate developmental aging and photoperiodic signals into the FT-FD module in Arabidopsis flowering. Mol. Plant 9, 1647-1659 (2016).

75. Padmanabhan, M. S. et al. Novel positive regulatory role for the SPL6 transcription factor in the N TIR-NB-LRR receptor-mediated plant innate immunity. PLoS Pathog. 9, e1003235 (2013).

76. $\mathrm{Xu}, \mathrm{P}$. et al. Virus infection improves drought tolerance. New Phytol. 180, 911-921 (2008).

77. Arshad, M., Feyissa, B. A., Amyot, L., Aung, B. \& Hannoufa, A. MicroRNA156 improves drought stress tolerance in alfalfa (Medicago sativa) by silencing SPL13. Plant Sci. 258, 122-136 (2017). 